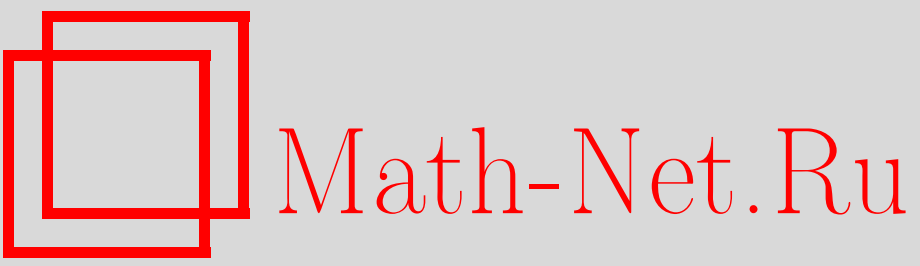

В. Ж. Сакбаев, О динамике множества состояний квантовой системы с вырожденным гамильтонианом, Вестн. Сам. гос. техн. ун-та. Сер. Физ.-мат. науки, 2011, выпуск 2(), 200-220

DOI: https://doi.org/10.14498/vsgtu873

Использование Общероссийского математического портала Math-Net.Ru подразумевает, что вы прочитали и согласны с пользовательским соглашением

http: //www. mathnet.ru/rus/agreement

Параметры загрузки:

IP : 54.162 .127 .20

26 апреля 2023 г., 16:18:48 
УДК 519.98

\title{
О ДИНАМИКЕ МНОЖЕСТВА СОСТОЯНИЙ КВАНТОВОЙ СИСТЕМЫ С ВЫРОЖДЕННЫМ ГАМИЛЬТОНИАНОМ
}

\author{
В. ЖК. Сакбаев ${ }^{1,2}$ \\ 1 Московский физико-технический институт (государственный университет), \\ 141700, Московская обл., Долгопрудный, Институтский переулок, 9. \\ 2 Российский университет дружбы народов, \\ 117198, Москва, ул. Миклухо-Маклая, 6. \\ E-mail: fumi2003@mail.ru \\ С задачей Коши для уравнения Шрёдингера с разрывными вырожсдающими- \\ ся коэффициентами в рамках метода эллиптической регуляризаиии связана \\ последовательность регуляризованных задач Коши и соответствующих регу- \\ ляризованных квантовых состояний. Получены необходимые и достаточные \\ условия сходимости последовательности регуляризованных динамических по- \\ лугрупп. В случае отсутствия сходимости расходящаяся последовательность \\ квантовых состояний изучается как случайный прощесс, определённый на из- \\ меримом пространстве параметров регуляризаиии с конечно-аддитивной ме- \\ рой. Математическое ожидание рассматриваемого процесса задаёт усреднен- \\ ную траекторию в пространстве квантовых состояний. Определены условия на \\ конечно-аддитивную меру, благодаря которым усредненная траектория опреде- \\ ляется по её значениям в два различных момента времени с помощью решения \\ вариационных задач.
}

Ключевые слова: конечно-аддитивная мера, случайный процесс, квантовое состояние, динамическая полугруппа, наблюдаемость.

Введение. Вырождение гамильтониана квантовой системы на некотором подмножестве координатного пространства может привести к некорректности задачи Коши для уравнения Шрёдингера [1,2]. Для определения динамики системы с вырожденным гамильтонианом как в классической [3], так и в квантовой [4] механике используется метод регуляризации вырожденного гамильтониана. Динамическое преобразование пространства состояний системы с вырожденным гамильтонианом определяется как предел последовательности регуляризованных динамических полугрупп, задаваемых регуляризованными гамильтонианами. Процедурам предельного перехода для последовательностей динамических систем, определяемых дифференциальными уравнениями с малым параметром, и усреднения таких систем посвящена работа Н. Н. Боголюбова [5]. Настоящая работа посвящается применению операции усреднения по конечно-аддитивным мерам к квантовым системам с гамильтонианами, содержащими малый параметр.

Последовательности регуляризованных гамильтонианов с малым параметром, аппроксимирующей вырожденный оператор, поставлено в соответствие однопараметрическое семейство усреднённых динамических преобразований множества квантовых состояний, которое служит предельной точкой последовательности регуляризованных динамических полугрупп. Установлено, что полученное семейство усреднённых преобразований не обладает полугрупповым свойством, но траектория семейства усреднённых преобразований однозначно определяется по своим значениям в два различных момента вре-

Всеволод Жанович Сакбаев (д.ф.-м.н., доц.), доцент, каф. высшей математики혁 доцент, каф. дифференциальных уравнений и математической физики ${ }^{2}$. 
мени посредством решения вариационных задач. Кроме того, усреднённые преобразования переводят чистые квантовые состояния в состояния общего вида [6]. В работе исследована структура множества квантовых состояний и установлена представимость произвольного состояния в виде интеграла по множеству векторных состояний.

В работе изучается задача Коши для уравнения Шрёдингера с вырожденным гамильтонианом

$$
\begin{gathered}
i \frac{d}{d t} u(t)=\mathbf{L} u(t), \quad t>0, \\
u(+0)=u_{0}, \quad u_{0} \in H=L_{2}(\mathbb{R}),
\end{gathered}
$$

где $\mathbf{L}$ - симметрический плотно определённый оператор в пространстве $H$, заданный линейным дифференциальным выражением второго порядка с неотрицательной характеристической формой. Поскольку вырождение гамильтониана $\mathbf{L}$ может привести к некорректности задачи Коши (1), (2), то в рамках метода эллиптической регуляризации исследуется последовательность регуляризованных задач Коши с начальным условием (2) для уравнений Шрёдингера

$$
i \frac{d}{d t} u(t)=\mathbf{L}_{\varepsilon} u(t), \quad t>0, \quad \varepsilon \in E \equiv(0,1),
$$

где при каждом $\varepsilon \in E$ оператор $\mathbf{L}_{\varepsilon}$ самосопряжен и равномерно эллиптичен, а при $\varepsilon \rightarrow 0$ последовательность операторов $\left\{\mathbf{L}_{\varepsilon}\right\}$ аппроксимирует оператор $\mathbf{L}$.

В качестве примера вырожденного симметрического оператора может быть предложен оператор $\mathbf{L}$, заданный на максимальной области определения в пространстве $H=L_{2}(\mathbb{R})$ дифференциальным выражением второго порядка

$$
\mathbf{L} v(x)=\frac{\partial}{\partial x}\left(g(x) \frac{\partial}{\partial x} v(x)\right)+\frac{i}{2}\left(a(x) \frac{\partial}{\partial x} v(x)+\frac{\partial}{\partial x}(a(x) v(x))\right),
$$

где функция $g=\chi_{R_{-}}$есть характеристическая функция полупрямой $\mathbb{R}_{-}=$ $=(-\infty, 0)$, а вещественнозначная функция $a=\alpha \chi_{R_{+}}$пропорциональна характеристической функции полупрямой $\mathbb{R}_{+}=(0,+\infty)$. Примером регуляризации служит семейство операторов $\mathbf{L}_{\varepsilon}$, заданных на максимальной области определения в пространстве $H$ дифференциальным выражением вида (4) с функцией $g_{\varepsilon}=g+\varepsilon, \varepsilon \in E$, вместо функции $g$ (см. [2]). При каждом значении $\varepsilon \in E$ оператор $\mathbf{L}_{\varepsilon}$ генерирует регуляризованную унитарную полугруппу $\mathbf{U}_{\mathbf{L}_{\varepsilon}}(t)=e^{-i \mathbf{L}_{\varepsilon} t} 4, t>0$, в пространстве $H$ и определяет регуляризованное решение $u_{\varepsilon}\left(t, u_{0}\right)=\mathbf{U}_{\mathbf{L}_{\varepsilon}}(t) u_{0}$.

$\mathrm{B}$ настоящей работе исследуется случай (подобный приведённому в примере после равенства (4)), когда оператор $\mathbf{L}$ является максимальным симметрическим оператором, а оператор $-i \mathbf{L}$ не является генератором сжимающей полугруппы, что в терминах индексов дефекта оператора $\mathbf{L}$ эквивалентно условиям

$$
\operatorname{dim}\left(\operatorname{Ker}\left(\mathbf{L}^{*}-i \mathbf{I}\right)\right)=0, \quad \operatorname{dim}\left(\operatorname{Ker}\left(\mathbf{L}^{*}+i \mathbf{I}\right)\right)>0 .
$$

В работе [2] доказано, что при условии (5) оператор $i \mathbf{L}$ генерирует изометрическую полугруппу $\mathbf{U}_{-\mathbf{L}}(t)=e^{i \mathbf{L} t} t>0$, в пространстве $H$ и сопряженный оператор $-i \mathbf{L}^{*}$ генерирует сжимающую полугруппу $\mathbf{U}_{\mathbf{L}^{*}}(t)=e^{-i \mathbf{L}^{*} t}, t>0$ и справедливо 
ПРЕДЛОЖенИЕ 1. Пусть выполнены условия (5). Тогда при каждом $t>0$ пространство $H$ допускает ортогональное разложсение $H=H_{0}(t) \oplus H_{1}(t)$, где $H_{0}(t)=\overline{\operatorname{Im}(\mathbf{U}-\mathbf{L}(t))}$ и $H_{1}(t)=\operatorname{Ker}\left(\mathbf{U}_{\mathbf{L}^{*}}(t)\right)$, таким образом, что для любого $u_{0} \in H_{1}(t)$ последовательность регуляризованных решений $\left\{u_{\varepsilon}\left(t, u_{0}\right)\right\}$ сходится при $\varepsilon \rightarrow 0$ слабо в $H \kappa$ нулю, а при любом $u_{0} \in H_{0}(t)$ справедливо равенство $\lim _{\varepsilon \rightarrow 0}\left\|u_{\varepsilon}\left(t, u_{0}\right)-\mathbf{U}_{\mathbf{L}^{*}}(t) u_{0}\right\|_{H}=0$.

Фильтры, ультрафильтры и двузначные меры. Пусть $\mathbb{N}$ - множество натуральных чисел, $2^{\mathbb{N}}-\sigma$-алгебра всех его подмножеств, $B\left(\mathbb{N}, 2^{\mathbb{N}}\right)=l_{\infty}$ и $\mathrm{ba}\left(\mathbb{N}, 2^{\mathbb{N}}\right)=l_{\infty}^{*}$, через са $\left(\mathbb{N}, 2^{\mathbb{N}}\right)$ и $\mathrm{pba}\left(\mathbb{N}, 2^{\mathbb{N}}\right)$ обозначим соответственно счётно-аддитивную и чисто конечно-аддитивную части пространства $l_{\infty}^{*}$ $\left(\mathrm{ca}\left(\mathbb{N}, 2^{\mathbb{N}}\right)=l_{1}\right)($ см. [8]). Через $V(\mathbb{N})$ обозначим пересечение единичной сферы пространства $l_{\infty}^{*}$ с конусом его неотрицательных элементов, а через $W(\mathbb{N})-$ пересечение $\operatorname{pba}\left(\mathbb{N}, 2^{\mathbb{N}}\right) \bigcap V(\mathbb{N})$, т. е. множество таких неотрицательных нормированных мер на $\mathbb{N}$, которые принимают нулевое значение на любом конечном подмножестве.

Согласно результатам [9] множества $V(\mathbb{N})$ и $W(\mathbb{N})$ есть выпуклые компактные в $*$-слабой топологии пространства пространства $l_{\infty}^{*}$, множества крайних точек которых составляют соответственно множества $V_{0}(\mathbb{N})=\operatorname{Extr}(V(\mathbb{N}))$ и $W_{0}(\mathbb{N})=\operatorname{Extr}(W(\mathbb{N}))$ мер, принимающих на алгебре $2^{\mathbb{N}}$ лишь два значения: 0 или 1 . Таким образом, для каждой меры $\mu \in V_{0}(\mathbb{N})$ алгебра $2^{\mathbb{N}}$ представима в виде объединения двух непересекающихся множеств $S_{\mu}(0)$ и $S_{\mu}(1)$, определяемых равенствами $S_{\mu}(s)=\mu^{-1}(s)$. Следовательно, для любой меры $\mu \in V_{0}(\mathbb{N})$ совокупность $S_{\mu}(1)$ элементов алгебры $2^{\mathbb{N}}$ образует ультрафильтр [8], который будем обозначать через $\digamma_{\mu}$. Тогда для меры $\mu \in W(\mathbb{N})$ ультрафильтр $\digamma_{\mu}$ является неглавным (т. е. пересечение всех множеств из $\digamma_{\mu}$ пусто), а для меры $\mu \in V\left(\mathbb{N}, 2^{\mathbb{N}}\right) \bigcap \operatorname{ca}\left(\mathbb{N}, 2^{\mathbb{N}}\right)$ - главным. Причём различным мерам $\mu, \nu \in W_{0}(\mathbb{N})$ соответствуют различные ультрафильтры $\digamma_{\mu}$ и $\digamma_{\nu}$, такие, что найдутся непересекающиеся подмножества $\mathbb{N}_{\alpha} \in \digamma_{\mu}$ и $\mathbb{N}_{\beta} \in \digamma_{\nu}$.

Наоборот, если задан некоторый ультрафильтр $\digamma$, то равенство $\mu\left(\mathbb{N}_{\alpha}\right)=$ $=1 \forall \mathbb{N}_{\alpha} \in \digamma$ определяет меру $\mu_{\digamma} \in V_{0}(\mathbb{N})$. При этом, если два ультрафильтра $\digamma$, J различны, то они содержат непересекающиеся подмножества $F_{\alpha} \in \digamma$ и $G_{\beta} \in \mathbb{I}$, а соответствующие им меры $\mu_{\digamma}, \mu_{\beth} \in V_{0}(\mathbb{N})$ различны. Следовательно, можно отождествить чисто конечно-аддитивные двузначные меры $\mu \in V_{0}(\mathbb{N})$ и ультрафильтры подмножеств $\digamma \in 2^{\mathbb{N}}$. Если $\mu=\mu_{\digamma}$, то будем говорить, что ультрафильтр $\digamma$ является носителем меры $\mu \in V_{0}(\mathbb{N})$.

Квантовые состояния. Пусть $B(H)$ - банахово пространство линейных ограниченных операторов в пространстве $H$ и $B^{*}(H)$ - его сопряженное, а $B_{*}(H)$ - предсопряженное пространство ядерных операторов. Далее через $\Sigma(H)=S_{1}\left(B^{*}(H)\right) \bigcap B_{+}^{*}(H)$ обозначается множество квантовых состояний части единичной сферы пространства $B^{*}(H)$ (здесь и далее через $S_{1}(X)$ обозначается единичная сфера в банаховом пространстве $X$ ), лежащей в положительном конусе $B_{+}{ }^{*}(H)$ функционалов из $B^{*}(H)$, принимающих неотрицательные значения на положительных операторах из пространства $B(H)$ (см. [6]). Через $\Sigma_{n}(H)$ обозначим множество нормальных квантовых состояний - функционалов из $\Sigma(H)$, непрерывных не только по норме, но и в сильной операторной топологии. Через $\Sigma_{p}(H)$ будем обозначать множество чи- 
стых квантовых состояний - множество крайних точек пространства $\Sigma_{n}(H)$, задаваемых проекторами на одномерные подпространства пространства $H$ (см. [6]). Чистое квантовое состояние, отвечающее проектору на единичный вектор $u \in H$, задаёт на пространстве $B(H)$ линейный непрерывный функционал $\rho_{u}:\left\langle\rho_{u}, \mathbf{A}\right\rangle=(u, \mathbf{A} u)_{H}$. Далее будем отождествлять чистые состояния и одномерные ортогональные проекторы, а также нормальные состояния и неотрицательные ядерные операторы с единичной нормой в $B_{*}(H)$.

Согласно теореме Крейна-Мильмана всякое компактное выпуклое множество в линейном топологическом пространстве совпадает с замыканием выпуклой оболочки множества своих крайних точек. Так, множество крайних точек совокупности нормальных состояний $\Sigma_{n}(H)$ составляет множество $\Sigma_{p}(H)$ чистых векторных состояний. Действительно, всякое векторное состояние является, очевидно, крайней точкой множества $\Sigma_{n}(H)$, а любое другое состояние из множества $\Sigma_{n}(H)$ является выпуклой комбинацией двух ортогональных нормальных состояний с ненулевыми коэффициентами. Следовательно, множество $\Sigma_{n}(H)$ совпадает с замыканием выпуклой оболочки множества $\Sigma_{p}(H)$ в топологии нормы пространства $B_{*}(H)$.

Teopema E (Теорема 6, гл. 2, Стр. 136 [10]). Множество $\Sigma(H)$ совпадает с замыканием выпуклой оболочки множества $\Sigma_{p}(H)$ в $*$-слабой топологии пространства $B^{*}(H)$.

На основании приведённой теоремы получим разложение произвольного квантового состояния (не обязательно нормального) в интеграл по множеству чистых состояний. Полученное представление состояния общего вида является аналогом спектрального разложения нормального состояния и аналогом разложения Глаубера-Сударшана нормального состояния (см. [11]).

Теорема 1. Пусть $H$ - сепарабельное гилъбертово пространство. Тогда существует такая последовательность нормальных состояний $\left\{\rho_{m}\right\}$, что для каждого состояния $\rho \in \Sigma(H)$ найдётся мера $\mu \in W_{0}(\mathbb{N})$ такая, ито

$$
\rho=\int_{\mathbb{N}} \rho_{k} d \mu(k)
$$

которое понимается в смысле Петтиса:

$$
\langle\rho, \mathbf{A}\rangle=\int_{\mathbb{N}}\left\langle\rho_{k}, \mathbf{A}\right\rangle d \mu(k) \quad \forall \mathbf{A} \in B(H)
$$

Доказательство. Пусть $\Omega_{v}$ есть множество выпуклых комбинаций чистых состояний вида $\sum_{j=1}^{m} \lambda_{j} \rho_{u_{j}}$, где $u_{1}, \ldots, u_{m} \in S_{1}(H), \lambda_{1}, \ldots, \lambda_{m} \in[0,1]$ и $\sum_{j=1}^{m} \lambda_{j}=1$. Через $\Omega_{u}$ обозначим множество выпуклых комбинаций попарно ортогональных чистых состояний вида $\sum_{j=1}^{m} \lambda_{j} \rho_{u_{j}}$, где $u_{1}, \ldots, u_{m} \in$ $S_{1}(H)$ - некоторая конечная ортонормированная система векторов, $\lambda_{1}, \ldots$, $\lambda_{m} \in[0,1]$ и $\sum_{j=1}^{m} \lambda_{j}=1$. Очевидно, что $\Omega_{u} \subset \Omega_{v}$, но поскольку любой элемент $\rho \in \Omega_{v}$ есть положительный самосопряженный оператор конечного ранга с единичным следом, то в силу спектральной теоремы $\rho \in \Omega_{u}$ и, следовательно, $\Omega_{v}=\Omega_{u}$. 
Поскольку пространство $H$ сепарабельно, то существует счётное всюду плотное на единичной сфере $S_{1}(H)$ подмножество векторов единичной сферы $\left\{u_{j}, j \in \mathbb{N}\right\}$. Множество $\Omega_{u}^{c}$ линейных комбинации чистых состояний $\rho_{u_{j}}$ с неотрицательными рациональными коэффициентами образует счетное подмножество множества $\Omega_{u}$.

Множество $\Omega_{u}^{c}$ плотно в $\Omega_{u}(H)$ по норме пространства $B_{*}(H)$, что следует из спектральной теоремы для ядерного оператора и всюду плотности множества $\left\{u_{j}, j \in \mathbb{N}\right\}$ на единичной сфере $S_{1}(H)$. Пусть последовательность $\left\{\rho_{k}\right\}$ задаёт некоторую нумерацию элементов множества $\Omega_{u}^{c}$. Покажем, что эта последовательность нормальных состояний является искомой.

Согласно теореме Е множество $\Sigma(H)$ совпадает с замыканием в $*$-слабой топологии множества $\Omega_{v}$ и, следовательно, множества $\Omega_{u}^{c}$. Поэтому для любого элемента $\rho \in \Sigma(H)$, любого конечного набора операторов $\mathbf{A}_{1}, \ldots, \mathbf{A}_{m} \in B(H)$ и любого $\varepsilon>0$ найдётся такое непустое подмножество $\mathbb{N}_{\varepsilon, \mathbf{A}_{1}, \ldots, \mathbf{A}_{m}}(\rho) \in 2^{\mathbb{N}}$, что для любого $k \in \mathbb{N}_{\varepsilon}, \mathbf{A}_{1}, \ldots, \mathbf{A}_{m}(\rho)$ выполняются неравенства $\left|\left\langle\rho-\rho_{k}, \mathbf{A}_{j}\right\rangle\right|<\varepsilon$, $j=1,2, \ldots, m$. Причём если $\varepsilon>0$, а $A_{1}, \ldots, A_{k}$ и $A_{k+1}, \ldots, A_{s}$ - два различных набора операторов, то множество $\mathbb{N}_{\varepsilon}, A_{1}, \ldots, A_{k}, A_{k+1}, \ldots, A_{s}$, во-первых, непусто в силу теоремы Е, и, во-вторых, содержится в множествах $\mathbb{N}_{\varepsilon}, A_{1}, \ldots, A_{k}$

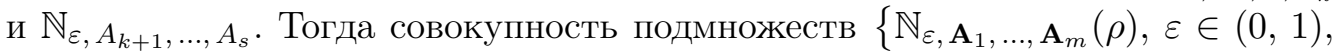
$\left.m \in \mathbb{N}, \mathbf{A}_{j} \in B(H) \forall j \in\{1,2, \cdots\}\right\}$ образует базу некоторого фильтра $\digamma_{0}$. Пусть ультрафильтр $\digamma$ мажорирует фильтр $\digamma_{0}$. Следовательно, для меры $\mu_{\digamma} \in W_{0}(\mathbb{N})$ выполнено (6), что и доказывает теорему 1.

Теорема 2. Пусть $H$-сепарабельное гилъбертово пространство. Для всякого состояния $\rho \in \Sigma(H)$ существует такая последовательность единичных векторов $\left\{u_{k}\right\}$ пространства $H$ и такая неотричательная нормированная мера $\mu \in \mathrm{ba}(\mathbb{N})$, что выполнено равенство

$$
\rho=\int_{\mathbb{N}} \rho_{u_{k}} d \mu(k),
$$

которое понимается в смысле Петтиса.

Доказ ательство. Пусть $\left\{u_{k}\right\}$ - последовательность единичных векторов гильбертова пространства $H$ со всюду плотным множеством значений на единичной сфере $S_{1}(H)$.

Для каждого оператора $\mathbf{A} \in B(H)$ по последовательности $\left\{u_{k}\right\}$ равенствами $a_{k}=\left(u_{k}, \mathbf{A} u_{k}\right), k \in \mathbb{N}$, определяется числовая последовательность $\left\{a_{k}\right\}=\alpha(\mathbf{A}) \in l_{\infty}$. Последовательность $\left\{\rho_{j}\right\}$, существование которой устанавливает теорема 1 , ставит в соответствие каждому оператору $\mathbf{A} \in B(H)$ числовую последовательность $\left\{b_{j}\right\}=\beta(\mathbf{A}) \in l_{\infty}: b_{j}=\left\langle\rho_{j}, \mathbf{A}\right\rangle$. Отображение $\beta: B(H) \rightarrow l_{\infty}$ линейно, непрерывно и изометрично, а отображение $\alpha: B(H) \rightarrow l_{\infty}$ линейно, непрерывно, изометрично и инъективно. Действительно, если ограниченные операторы $\mathbf{A}_{1}, \mathbf{A}_{2}$ различны, то найдётся единичный вектор пространства $H$, на котором различаются их квадратичные формы. Так как множество значений последовательности $\left\{u_{k}\right\}$ всюду плотно в $H$, то найдётся такое $k$, что $\left(u_{k}, \mathbf{A}_{1} u_{k}\right) \neq\left(u_{k}, \mathbf{A}_{2} u_{k}\right)$ и, следовательно, $\alpha\left(\mathbf{A}_{1}\right) \neq \alpha\left(\mathbf{A}_{2}\right)$. А в силу определения нормы оператора равенство $\|\mathbf{A}\|_{B(H)}=$ $=\|\alpha(\mathbf{A})\|_{l_{\infty}}$ справедливо при любом $\mathbf{A} \in B(H)$. Пусть $\Lambda=(\alpha)^{-1}$ - обратный оператор к отображению $\alpha$, заданный на образе $\operatorname{Im}(\alpha)$ оператора $\alpha$ в про- 
странстве $l_{\infty}$. Тогда отображение $\Lambda$ линейно, биективно, изометрично и, следовательно, непрерывно.

Согласно теореме 1 существует такая мера $\mu \in W_{0}(\mathbb{N})$, что

$$
\rho(A)=\int_{\mathbb{N}}\left\langle\rho_{j}, \mathbf{A}\right\rangle d \mu(j)
$$

и, следовательно, $\rho(\mathbf{A})=\langle\mu, \beta(\mathbf{A})\rangle$ в силу определения отображения $\beta$.

Определим меру $m$ как линейный непрерывный функционал на пространстве $l_{\infty}$, который на линейном многообразии $\operatorname{Im}(\alpha)$ задается равенством $m=$ $=\mu \circ \beta \circ \Lambda$ как композиция линейных ограниченных отображений. (С линейного многообразия $\operatorname{Im}(\alpha)$ на пространство $l_{\infty}$ может быть продлен какимлибо образом по теореме Хана-Банаха. Тот факт, что единичная функция 1 входит в многообразие $\operatorname{Im}(\alpha)$ и $m(\mathbf{1})=1$, обеспечивает неотрицательность и нормировку любого такого продолжения.)

Поэтому если $m=\mu \circ \beta \circ \Lambda$, то

$$
\int_{\mathbb{N}}\left\langle\rho_{j}, \mathbf{A}\right\rangle d \mu(j)=\int_{\mathbb{N}}\left\langle\rho_{u_{k}}, \mathbf{A}\right\rangle d m .
$$

Ибо

$$
\begin{aligned}
\rho(A)=\int_{\mathbb{N}}\left\langle\rho_{j}, \mathbf{A}\right\rangle d \mu(j)=\langle\mu, \beta(\mathbf{A})\rangle= \\
\quad=\langle\mu, \beta(\Lambda(\alpha \mathbf{A}))\rangle=\langle m, \alpha(\mathbf{A})\rangle=\int_{\mathbb{N}}\left\langle\rho_{u_{k}}, \mathbf{A}\right\rangle d m .
\end{aligned}
$$

Следовательно, для любого $\rho \in \Sigma(H)$ найдётся мера $m \in V(\mathbb{N})$ такая, что выполняется (7).

Таким образом, произвольное квантовое состояние $r$ из множества $\Sigma(H)$ допускает вид разложения в интеграл (7) по векторным состояниям по конечно-аддитивной мере $\mu$ на множестве $\Sigma_{p}(H)$. Заметим, что состояния указанного вида изучались в работах Диксмье [12] по обобщению понятия следа, в работе Шриниваза [13] по постулатам редукции в теории квантовых измерений, а также к указанному виду относятся состояния Кубо-МартинаШвингера $[4,10]$. В указанных исследованиях квантовое состояние, не являющееся нормальным, представлено как инвариантное среднее от последовательности чистых векторных состояний, то есть как интеграл вида (7) по мере $\mu$, удовлетворяющей условиям инвариантности относительно некоторой группы преобразований (например, сдвига). Мы отказываемся от последнего требования, поскольку ставим цель определить не операцию обобщенного следа (не зависящую от выбора базиса), а некоторое конкретное состояние на $B(H)$ (для которого не все базисы в $H$ равноправны).

Именно квантовые состояния, представляемые разложением вида (7), возникают в работе [14] при описании множества предельных точек последовательности регуляризованных состояний в $*$-слабой топологии пространства $B^{*}(H)$. Чтобы проанализировать свойства расходящейся ограниченной последовательности решений $\left\{u_{\varepsilon}\left(t, u_{0}\right)\right\}$, рассмотрим соответствующую ей последовательность регуляризованных операторов плотности $\left\{\rho_{\varepsilon}\left(t, \rho_{u_{0}}\right), t>0\right.$, 
$\varepsilon \in E, \varepsilon \rightarrow 0\}$, где при каждом $\varepsilon \in E$ функция $\rho_{\varepsilon}\left(t, \rho_{0}\right): \mathbb{R}_{+} \rightarrow B^{*}(H)$ определена равенством $\left\langle\rho_{\varepsilon}\left(t, \rho_{0}\right), \mathbf{A}\right\rangle=\left\langle\rho_{0}, \mathbf{U}_{\mathbf{L}_{\varepsilon}}(-t) \mathbf{A U}_{\mathbf{L}_{\varepsilon}}(t)\right\rangle$. Исследуем также последовательность регуляризованных динамических полугрупп $\left\{T_{\varepsilon}(t), t>\right.$ $0\}_{\varepsilon \in E, \varepsilon \rightarrow 0}$ в пространстве $B^{*}(H)$, каждая из которых определяется равенством $T_{\varepsilon}(t) \rho_{0}=\rho_{\varepsilon}\left(t, \rho_{0}\right)$.

В [7] установлено, что необходимым и достаточным условием секвенциальной компактности множества значений последовательности $\left\{\rho_{\varepsilon}\left(t, \rho_{u_{0}}\right)\right\}$ в *-слабой топологии пространства $B^{*}(H)$ является включение $u_{0} \in H_{0}(t)$. В случае $u_{0} \notin H_{0}(t)$ определим для каждого оператора $\mathbf{A} \in B(H)$ множество

$$
G_{A}\left(t, u_{0}\right)=\left\{r \in \Sigma(H):\langle r, \mathbf{A}\rangle \in L s_{\varepsilon \rightarrow 0}\left\langle\rho_{\varepsilon}\left(t, u_{0}\right), \mathbf{A}\right\rangle\right\},
$$

где $L s_{\varepsilon \rightarrow 0}\left\langle\rho_{\varepsilon}\left(t, u_{0}\right), \mathbf{A}\right\rangle$ - множество частичных пределов числовой функции $\left\langle\rho_{\varepsilon}\left(t, u_{0}\right), \mathbf{A}\right\rangle, \varepsilon \in E$, при $\varepsilon \rightarrow 0$. Согласно [7], множество

$$
\Phi\left(t, u_{0}\right)=\bigcap_{\mathbf{A} \in B(H)} G_{\mathbf{A}}\left(t, u_{0}\right)
$$

выпукло, замкнуто в $*$-слабой топологии пространства $B^{*}(H)$ и удовлетворяет равенству $\left\langle\Phi\left(t, u_{0}\right), \mathbf{A}\right\rangle=L s_{\varepsilon \rightarrow 0}\left\langle\rho_{\varepsilon}\left(t, u_{0}\right), \mathbf{A}\right\rangle$ при любом $\mathbf{A} \in B(H)$.

Случайный процесс. Рассмотрим $\sigma$-алгебру $2^{E}$ всех подмножеств множества параметров регуляризации $E$ и обозначим через $W(E)$ совокупность всех конечно-аддитивных мер, заданных на измеримом пространстве $\left(E, 2^{E}\right)$, неотрицательных, нормированных и удовлетворяющих условию: мера любого множества $A \in 2^{E}$, замыкание которого не содержит предельной точки $e^{*}=0=\inf E$, равна нулю. Множество $W(E)$ есть непустое выпуклое подмножество в пересечении конуса неотрицательных элементов с единичной сферой в банаховом пространстве $\mathrm{ba}\left(E, 2^{E}\right)$ конечно-аддитивных мер на измеримом пространстве $\left(E, 2^{E}\right)$ (см. [15]). Множество $W(E)$, очевидно, замкнуто в $*$-слабой топологии пространства ba $(E)$. Согласно теореме 33 [15] множество крайних точек $\operatorname{Extr}(W(E))$ множества $W(E)$ представляет собой подмножество $W_{0}(E)$ двузначных мер $\mu \in W(E)$, принимающих только значения 0 и 1 на $\sigma$-алгебре $2^{E}$.

В работе [16] расходящаяся последовательность $\left\{\rho_{\varepsilon}\left(t, u_{0}\right)\right\}$ исследована как случайный процесс - семейство измеримых отображений (параметризованных переменной $t>0)$ измеримого пространства $\left(E, 2^{E}\right)$ с мерой $\mu \in W(E)$ в измеримое пространство - банахово пространство $B^{*}(H)$ с алгеброй цилиндрических подмножеств $\mathrm{Cyl}_{Y_{*}}(Y)$, порождённой конечными наборами функционалов из $B(H)$. Каждое семейство отображений $\left\{\rho_{\varepsilon}\left(t, u_{0}\right)\right\}$ удовлетворяет условию

$$
\lim _{t \rightarrow+0} \sup _{\mathbf{A} \in B(H),\|\mathbf{A}\|_{\mathbf{B}(\mathbf{H})}=\mathbf{1}}\left|\rho_{\varepsilon}\left(t, u_{0}\right)(\mathbf{A})-\rho_{u_{0}}(\mathbf{A})\right|=0
$$

при любом $\varepsilon \in E$ (т. е. процесс $\rho_{\varepsilon}\left(t, u_{0}\right)$ начинается в точке $\rho_{u_{0}}$ пространства $\left.B^{*}(H)\right)$. Математическое ожидание случайной величины $\rho_{\varepsilon}\left(t, \rho_{0}\right)$ при каждом $t>0$ определяется как интеграл Петтиса:

$$
\rho^{\mu}\left(t, \rho_{0}\right)=\int_{E} \rho_{\varepsilon}\left(t, \rho_{0}\right) d \mu
$$


то есть линейный непрерывный функционал $\rho^{\mu}\left(t, \rho_{0}\right)$ на пространстве $B(H)$ задан равенством

$$
\left\langle\rho^{\mu}\left(t, \rho_{0}\right), \mathbf{A}\right\rangle=\int_{E}\left\langle\rho_{0}, \mathbf{U}_{\mathbf{L}_{\varepsilon}(t)^{*}} \mathbf{A} \mathbf{U}_{\mathbf{L}_{\varepsilon}(t)}\right\rangle
$$

В [14] каждой мере $\mu \in W(E)$ ставится в соответствие однопараметрическое семейство усреднённых динамических преобразований $T^{\mu}(t), t>0$, пространства $B^{*}(H)$ таких, что $T^{\mu}(t) \rho_{0}=\rho^{\mu}\left(t, \rho_{0}\right), \rho_{0} \in B^{*}(H)$. Очевидно, отображение $T^{\mu}(t)$ определено на пространстве $B^{*}(H)$, линейно и непрерывно, причём множество квантовых состояний $\Sigma(H)$ отображается им в себя. Нашей целью является изучение свойств (полугруппового свойств, свойств инъективности и сюръективности) однопараметрических семейств динамических преобразований $T^{\mu}(t), t>0$, пространства $B^{*}(H)$, анонсированных в работе [14].

Свойства усреднённых преобразований. Напомним, что оператор $\mathbf{L}$ удовлетворяет предположению (5). Пусть $t>0, H_{0}(t)=\overline{\operatorname{Im}\left(\mathbf{U}_{-\mathbf{L}}(t)\right)}$ и $H_{1}(t)=$ $=\operatorname{Ker}\left(\mathbf{U}_{\mathbf{L}^{*}}(t)\right)$. В работе [17] установлено, что при любом $\mu \in W(E)$ сужение $\left.T^{\mu}(t)\right|_{\Sigma\left(H_{0}(t)\right)}$ является биективным и даётся равенством $T^{\mu}(t) \rho_{0}=e^{-i \mathbf{L}^{*} t} \rho_{0} e^{i \mathbf{L} t}$, $\rho_{0} \in \Sigma\left(H_{0}(t)\right)$. Для описания свойств сужения $\left.T^{\mu}(t)\right|_{\Sigma\left(H_{1}(t)\right)}$ приведём доказанное в [17] утверждение. Напомним, что для любого $x \in H_{1}(t)$ и любого $h \in H$ справедливо равенство $\lim _{\varepsilon \rightarrow 0}\left(e^{-i \mathbf{L}_{\varepsilon} t} x, h\right)=0$.

Лемма 1. Пусть $t>0,\left\{e_{k}\right\}$-базис пространства $H_{1}(t) u\left\{\varepsilon_{k}\right\}-б е c$ конечно малая последовательность положительных чисел. Тогда каждому числу $m \in \mathbb{N}$ соответствует такая подпоследовательность $\left\{\varepsilon_{k}^{m}\right\}$ последовательности $\left\{\varepsilon_{k}\right\}$, что выполнены следующие утверждения:

1) для всех $k, l \in\{1,2, \ldots, m\}$ и любых $i, j \in \mathbb{N}, i \neq j$, выполнено

$$
\left|\left(\mathbf{U}_{\mathbf{L}_{\varepsilon_{i}^{m}}}(t) e_{k}, \mathbf{U}_{\mathbf{L}_{\varepsilon_{j}^{m}}}(t) e_{l}\right)\right|<2^{-(i+j+m)}
$$

2) для каждого $q \in\{1,2, \ldots, m\}$ последовательность $\left\{\varepsilon_{i}^{q}\right\}$ является подпоследовательностью $\left\{\varepsilon_{i}^{q-1}\right\}$, где $\varepsilon_{i}^{0}=\varepsilon_{i}, i \in \mathbb{N}$.

Доказ атель ств в. Пусть $\left\{\varepsilon_{k}\right\}$ - некоторая монотонно убывающая последовательность чисел множества $E$ и $E_{0}=\bigcup_{k \in \mathbb{N}} \varepsilon_{k}$. Докажем утверждение леммы с помощью двукратного применения метода математической индукции.

1. Пусть $m=1$. Выберем $\varepsilon_{1}^{1}=\varepsilon_{1}$ и положим $f_{1}^{1}=\mathbf{U}_{\varepsilon_{1}^{m 1}}(t) e_{1}$. Поскольку для любого вектора $x \in H_{1}(t)$ слабый предел при $\varepsilon \rightarrow 0$ обобщённой последовательности векторов $\left\{\mathbf{U}_{\varepsilon}(t) x, \varepsilon \in E, \varepsilon \rightarrow 0\right\}$ равен нулю, можно записать

$$
\lim _{\varepsilon \rightarrow 0}\left(f_{1}^{1}, \mathbf{U}_{\varepsilon}(t) e_{1}\right)=0 .
$$

Следовательно, существует $\varepsilon_{2}^{1} \in\left(0, \varepsilon_{1}^{1}\right)$ такое, что $\left|\left(f_{1}^{1}, f_{1}^{2}\right)\right| \leqslant 2^{-4}$, где $f_{1}^{2}=$ $=\mathbf{U}_{\varepsilon_{2}^{1}}(t) e_{1}$. Пусть определены числа $\varepsilon_{1}^{1}, \ldots, \varepsilon_{n}^{1}$ такие, что $0<\varepsilon_{n}^{1}<\ldots<\varepsilon_{1}^{1}$ 
и для любого набора чисел $i, j \in\{1,2, \ldots, n\}, j \neq i$, выполняется неравенство $\left|\left(f_{1}^{i}, f_{1}^{j}\right)\right| \leqslant 2^{-(i+j+1)}$, где $f_{1}^{j}=\mathbf{U}_{\varepsilon_{j}^{1}}(t) e_{1}$. В силу слабой сходимости к нулю вектор-функций $\mathbf{U}_{\varepsilon}(t) e_{1}, \varepsilon \in E$, при $\varepsilon \rightarrow 0$, найдётся такое число $\varepsilon_{n+1} \in\left(0, \varepsilon_{n}\right)$, что неравенство $\left|\left(f_{1}^{i}, f_{1}^{n+1}\right)\right| \leqslant 2^{-(i+n+1+1)}$, где $f_{1}^{n+1}=$ $=\mathbf{U}_{\varepsilon_{n+1}^{1}}(t) e_{1}$, выполняется для всех $i \in\{1,2, \ldots, n\}$. Согласно принципу математической индукции справедливо следующее утверждение: существует подпоследовательность $\left\{e_{n}^{1}\right\}$ последовательности $\left\{e_{n}\right\}$ такая, что совокупность векторов $\left\{f_{1}^{n}=\mathbf{U}_{\varepsilon_{n}^{1}}(t) e_{1}, n \in \mathbb{N}\right\}$ образует нормированную систему, квадратично близкую к ортогональной в том смысле, что $\left|\left(f_{1}^{j}, f_{1}^{i}\right)\right|<2^{-1-j-i}$ для любых $i, j \in \mathbb{N}, i \neq j$. Следовательно, утверждение леммы 1 верно при $m=1$.

2. Фиксируем некоторое число $m \in \mathbb{N}$. Предположим, что существует такая подпоследовательность $\left\{\varepsilon_{k}^{m}\right\}$ последовательности $\left\{\varepsilon_{k}\right\}$, что выполнены утверждения 1) и 2) леммы 1.

Тогда так же, как и в пункте 1 , методом математической индукции доказывается, что последовательность $\varepsilon_{k}^{m}$ имеет подпоследовательность $\left\{e_{k}^{m+1}\right\}$ такую, что выполнены следующие утверждения:

1) для всех $k, l \in\{1,2, \ldots, m+1\}$ и любых $i, j \in \mathbb{N}, i \neq j$, выполнено неравенство

$$
\left|\left(\mathbf{U}_{\mathbf{L}_{i}^{m+1}}(t) e_{k}, \mathbf{U}_{\mathbf{L}_{\varepsilon_{j}^{m+1}}}(t) e_{l}\right)\right|<2^{-(i+j+m+1)} ;
$$

2) для каждого $q \in\{1,2, \ldots, m+1\}$ последовательность $\left\{\varepsilon_{i}^{q}\right\}$ является подпоследовательностью $\left\{\varepsilon_{i}^{q-1}\right\}$, где $\varepsilon_{i}^{0}=\varepsilon_{i}, i \in \mathbb{N}$.

В силу принципа математической индукции справедливо утверждение леммы.

Совершенно аналогично доказывается

Лемма 2. Пусть $t_{s}(s \in \mathbb{N})$ - некоторая нумерация рациональных чисел $t>0 u\left\{\varepsilon_{k}\right\}$ - бесконечно малая последовательность со значениями в $E$. Пусть в пространствах $H_{1}\left(t_{s}\right)$ выбраны ортонормированные базисы $\left\{e_{k}(s)\right\}$. Тогда для любого $m \in \mathbb{N}$ существует подпоследовательность $\left\{e_{k}^{m}\right\}$ такая, что выполнены утверждения:

1) для всех $s, k, l \in\{1,2, \ldots, m\}$ и любых $i, j \in \mathbb{N}, i \neq j$, выполнено неравенство

$$
\left|\left(\mathbf{U}_{\mathbf{L}_{\varepsilon_{i}^{m}}}\left(t_{s}\right) e_{k}(s), \mathbf{U}_{\mathbf{L}_{\varepsilon_{j}^{m}}}\left(t_{s}\right) e_{l}(s)\right)\right|<2^{-(i+j+m)} ;
$$

2) для каждого $q \in\{1,2, \ldots, m\}$ последовательность $\left\{\varepsilon_{i}^{q}\right\}$ является подпоследовательностью $\left\{\varepsilon_{i}^{q-1}\right\}$, где $\varepsilon_{i}^{0}=\varepsilon_{i}, i \in \mathbb{N}$.

Следствие 1. Для любого числа $m \in \mathbb{N}$ существует положительная бесконечно малая последовательность $\left\{\varepsilon_{n}^{m}\right\}$ со значениями в множестве $E$ такая, что последовательность векторов $\left\{f_{p}^{n}(m)\right\}$, где $f_{p}^{n}\left(m, t_{s}\right)=\mathbf{U}_{\varepsilon_{n}^{m}}\left(t_{s}\right) e_{p}(s)$, $n \in \mathbb{N}$, образует нормированную систему векторов в пространстве $H$, квадратично близкую $к$ ортогональной и удовлетворяющую оченке

$$
\left|\left(f_{q}^{i}\left(m, t_{s}\right), f_{p}^{j}\left(m, t_{s}\right)\right)\right| \leqslant 2^{-2(i+j+m)} \sigma
$$


для всех $i \neq j$ и всех $s, p, q \in\{1,2, \ldots, m\}$.

Лемма 3. Пусть $\left\{e_{k}^{m}\right\}(m \in \mathbb{N})$ - семейство подпоследовательностей, существование которого утверждает лемма 2 , и пусть $E^{m}=\bigcup_{k=1}^{\infty} \varepsilon_{k}^{m}$. Тогда существует мера $\mu \in W(E)$ такая, что для любого $m \in \mathbb{N}$ выполнено равенство $\mu\left(E^{m}\right)=1$.

Доказ а тельств в. Определим функцию множества $G: S \rightarrow R$ на следующей совокупности $S$ подмножеств множества $E$. Положим $G=0$ на конечных множествах и $G=1$ на их дополнениях; $G=1$ на всех множествах $E_{m}$ и $G=0$ на их дополнениях. Тогда функционал $g$ определён на совокупности характеристических функций указанных подмножеств по следующему правилу: $g\left(\xi_{A}\right)=G(A), A \in S$. Обозначим через $L(S)$ линейную оболочку совокупности функций из $S$. Тогда $L(S)$ - линейное подпространство банахова пространства $B(E)$ ограниченных функций на множестве $E$ с sup-нормой, a $g$ - линейный функционал на линейном подпространстве $L(S)$, ограниченный единицей по норме. По теореме Хана-Банаха функционал $g$ допускает продолжение $\gamma$ на всё пространство $B(E)$ с сохранением нормы. Тогда $\gamma \in B^{*}(E), \gamma$ - неотрицательный линейный функционал с единичной нормой на банаховом пространстве $B(E)$, причём $\gamma\left(\xi_{E_{m}}\right)=1$ при любом $m \in \mathbb{N}$. Поэтому сужение $\mu$ функционала $\gamma$ на алгебру характеристических функций множеств из $2^{E}$ является неотрицательной нормированной конечно-аддитивной мерой на измеримом пространстве $\left(E, 2^{E}\right)$, причём $\mu\left(E_{m}\right)=1$ при любом $m \in \mathbb{N}$. Определённая выше мера $\mu$ принадлежит классу $W(E)$, поскольку мера дополнения любой окрестности предельной точки $\varepsilon^{*}=0$ равна нулю.

Для каждого $t>0$ и выбранного базиса в пространстве $H_{1}(t)$ обозначим через $\mathcal{M}(t)$ множество мер, удовлетворяющих условию леммы 3. Тогда из леммы 1 и результатов работы [17] вытекает

Теорема 3. Пусть $t>0 u \mu \in \mathcal{M}(t)$. Тогда образом выпуклого множества $\Sigma\left(H^{1}(t)\right)$ пространства $B^{*}(H)$ при отображсени $T^{\mu}(t)$ является выпуклое множество $T_{t}^{\mu}\left(\Sigma\left(H_{1}(t)\right)\right)$ пространства $B(H)^{*}$. Образ множества $\Sigma_{p}\left(H_{1}(t)\right)$ крайних точек множества $\Sigma_{n}\left(H_{1}(t)\right)$ совпадает с множеством крайних точек образа $T^{\mu}(t)\left(\Sigma_{n}\left(H_{1}(t)\right)\right)$ :

$$
T^{\mu}(t)\left(\operatorname{Extr}\left(\Sigma_{n}\left(H_{1}(t)\right)\right)\right)=\operatorname{Extr}\left(T^{\mu}(t)\left(\Sigma_{n}\left(H^{1}(t)\right)\right)\right) .
$$

Существует обратное $\left.\kappa T^{\mu}(t)\right|_{\Sigma_{p}\left(H_{1}(t)\right)}$ отображсение $\Lambda^{\mu}(t)$.

Наблюдаемость случайного процесса. В статье [17] исследуется вопрос об определении начального состояния $\rho_{0}$ процесса $\rho_{\varepsilon}\left(t, \rho_{0}\right)$ на измеримом пространстве $\left(E, 2^{E}, \mu\right)$ с мерой $\mu \in \mathcal{M}(t)$ по набору значений его математического ожидания $\rho^{\mu}\left(t, \rho_{0}\right)$ на различных подмножествах алгебры $B(H)$.

Пусть $\left\{e_{j}^{0}\right\}$ и $\left\{e_{j}^{1}\right\}$ - ортонормированные базисы в подпространствах $H_{0}(t)$ и $H_{1}(t)$ соответственно, а $\mu \in \mathcal{M}(t)$. Пусть $H_{0,1}^{m}(t)-m$-мерное подпространства в пространстве $H_{0,1}(t)$, натянутое на первые $m$ базисных векторов. Для каждого $v_{0} \in H_{0}^{m}(t)$ определим ортогональный проектор $\mathbf{P}_{\mathbf{U}_{\mathbf{L}^{*}}(t) v_{0}}$ (здесь и далее через $\mathbf{P}_{u}$ обозначается оператор ортогонального проектирования в пространстве $H$ на одномерную линейную оболочку вектора $u$ ), а для каждого вектора $v_{1} \in H_{1}^{m}(t)$ определим оператор $\mathbf{Q}_{m}\left(v_{1}\right)$ как предел (существующий в силу (8)) в сильной операторной топологии частичных сумм опера- 
торного ряда

$$
\mathbf{Q}_{m}\left(v_{1}\right)=\sum_{\varepsilon_{k}^{m} \in E^{m}} \mathbf{P}_{\mathbf{U}_{\mathbf{L}_{k}^{m}}(t) v_{1}}
$$

Пользуясь попарной почти ортогональностью в смысле (8) членов ряда (11), можно показать, что справедлива

Лемма 4. Для любого $m \in \mathbb{N}$ функиия $\mathbf{Q}_{m}(v), v \in S_{1}\left(H_{1}^{m}(t)\right)$ является непрерывным отображением $m$-мерной сберы $S_{1}\left(H_{1}^{m}(t)\right)$ в банахово пространство $B(H)$.

ЗАмечАниЕ. Напомним, что $S_{1}(H)$ есть единичная сфера в пространстве $H$.

Доказ а тель ст в о. Действительно, пусть $u, v \in S_{1}\left(H_{1}^{m}(t)\right)$ и $\xi \in S_{1}(H)$. Положим $\mathbf{U}_{\varepsilon_{j}^{m}(t)} H_{1}^{m}(t)=H_{1+j}^{m}(t)$. Согласно лемме 1 подпространства $H_{1+j}^{m}(t)$, $j=0,1,2, \ldots$, почти ортогональны, и для любого вектора $x \in H_{1+j}^{m}(t)$ справедлива оценка $\left\|\pi_{j}^{\perp} x\right\|_{H} \leqslant 2^{-m-j+1}\|x\|_{H}$, где $\pi_{j}^{\perp}$ - ортогональный проектор на линейную оболочку подпространств $H_{1+k}^{m}(t)$ при всех $k \in\{0 \bigcup \mathbb{N} \backslash\{j\}\}$. Через $\mathbf{P}_{j}^{m}$ обозначим ортогональные проекторы на подпространства $H_{1+j}^{m}(t)$ и положим $\xi_{j}^{m}=\mathbf{P}_{j}^{m} \xi$. Тогда $\sum_{j=0}^{\infty}\left\|\xi_{j}^{m}\right\|_{H}^{2} \leqslant\|\xi\|_{H}^{2}\left(1+2^{-m+2}\right)$.

Оценим норму вектора

$$
\begin{aligned}
\left(\mathbf{Q}_{m}(v)-\mathbf{Q}_{m}(u)\right) \xi=\sum_{k=1}^{\infty}\left(\mathbf{P}_{\mathbf{U}_{\varepsilon_{k}^{m}}(t) v}-\mathbf{P}_{\mathbf{U}_{\varepsilon_{k}^{m}}^{m}(t) u}\right) \xi= \\
=\sum_{k=1}^{\infty}\left(\mathbf{P}_{\mathbf{U}_{\varepsilon_{k}^{m}}(t) v}-\mathbf{P}_{\mathbf{U}_{\varepsilon_{k}^{m}}(t) u}\right) \xi_{k}^{m}
\end{aligned}
$$

Поскольку $\left\|\mathbf{P}_{u}-\mathbf{P}_{v}\right\|_{B(H)} \leqslant 2\|u-v\|_{H}$, можно записать

$$
\begin{aligned}
& \left\|\left(\mathbf{Q}_{v}(m)-\mathbf{Q}_{u}(m)\right) \xi\right\|_{H}^{2} \leqslant \sum_{k=1}^{\infty}\left\|\left(\mathbf{P}_{\mathbf{U}_{\varepsilon_{k}^{m}}(t) v}-\mathbf{P}_{\mathbf{U}_{\varepsilon_{k}^{m}}(t) u}\right) \xi_{k}^{m}\right\|_{H}^{2}\left(1+2^{-m+2}\right) \leqslant \\
& \leqslant 4\|v-u\|_{H}^{2}\left(1+2^{-m+2}\right) \sum_{k=1}^{\infty}\left\|\xi_{k}^{m}\right\|_{H}^{2} \leqslant 4\left(1+2^{-m+2}\right)^{2}\|u-v\|_{H}^{2}\|\xi\|_{H}^{2} .
\end{aligned}
$$

Следовательно, утверждение леммы справедливо.

Для любых $m \in \mathbb{N}, \rho \in \Sigma(H)$ и $t \in R$ определим следующие функционалы:

$$
\begin{gathered}
\varphi_{0}^{m, \rho, t}(v)=\left\langle\rho, \mathbf{P}_{\mathbf{U}_{\mathbf{L}^{*}}(t) v}\right\rangle, v \in S_{1}\left(H_{0}^{m}(t)\right) \\
\varphi_{1}^{m, \rho, t}(v)=\left\langle\rho, \mathbf{Q}_{m}(v)\right\rangle, v \in S_{1}\left(H_{1}^{m}(t)\right)
\end{gathered}
$$

Тогда при любых $m \in \mathbb{N}, s \in\{0,1\}$ и $\rho \in \Sigma(H)$ непрерывный функционал $\varphi_{s}^{m, \rho, t}$ достигает максимума на некотором векторе $v_{s}^{m} m$-мерной сферы $S_{1}\left(H_{s}^{m}(t)\right)$. 
В разделе 4 работы [17] доказано, что для любых $\rho_{u_{0}} \in \Sigma_{p}(H)$, любых $m \in \mathbb{N}$ и $t>0$ справедливо равенство

$$
\varphi_{0}^{m, \rho^{\mu}\left(t, \rho_{u_{0}}\right), t}(v)=\left\|\left(u_{0}, v\right)\right\|^{2} .
$$

Поэтому последовательность $\left\{\sup _{v \in S_{1}\left(H_{0}^{m}(t)\right)} \varphi_{0}^{m, \rho^{\mu}\left(t, \rho_{0}\right), t}(v)\right\}$ монотонно возрастает и, следовательно, существует предел

$$
M_{0}(t) \equiv \lim _{m \rightarrow \infty} \sup _{v \in S_{1}\left(H_{0}^{m}(t)\right)} \varphi_{0}^{m, \rho^{\mu}\left(t, \rho_{0}\right), t}(v)=\left\|\mathbf{P}_{H_{0}(t)} u_{0}\right\|^{2} .
$$

В той же статье (см. лемму 4.3 [17]) установлено, что для любого чистого начального состояния $\rho_{0}=\rho_{u_{0}} \in \Sigma_{p}\left(H_{1}(t)\right)$ справедлива оценка

$$
\varphi_{1}^{m, \rho^{\mu}\left(t, \rho_{u_{0}}\right), t}(v)-\left|\left(u_{0}, v\right)\right|^{2}=\mathrm{o}(1) \quad \text { при } \quad m \rightarrow \infty
$$

и поэтому существует предел

$$
M_{1}(t) \equiv \lim _{m \rightarrow \infty} \sup _{v \in S_{1}\left(H_{1}^{m}(t)\right)} \varphi_{1}^{m, \rho^{\mu}\left(t, \rho_{0}\right), t}(v)=\left\|\mathbf{P}_{H_{s}(t)} u_{0}\right\|^{2}, \quad s \in\{0,1\} .
$$

Почти дословным повторением хода рассуждений работы [17] доказывается

Теорема 4. Пусть $t>0$ и начальное состояние $\rho_{0}$ удовлетворяет одному из двух условии: $\rho_{0} \in \Sigma_{n}\left(H_{0}(t)\right)$ либо $\rho_{0} \in \Sigma_{n}\left(H_{1}(t)\right)$. Пусть мера $\mu \in \mathcal{M}(t)$. Тогда

1) состояние $\rho_{0}$ является чистым, если и только если $\max \left\{M_{0}(t), M_{1}(t)\right\}=$ $=1$;

2) если начальное состояние $\rho_{0}$ является чистым состоянием в подпространстве $H_{0}(t)$ (или $\left.H_{1}(t)\right)$ и $\left\{v_{0}^{m}\right\}\left(\right.$ или $\left.\left\{v_{1}^{m}\right\}\right)$ есть последователь-

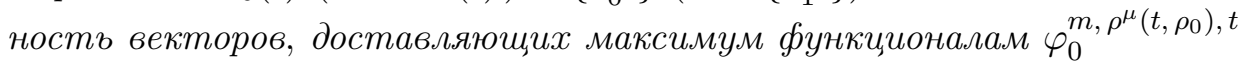
$\left(\right.$ или $\left.\varphi_{1}^{m, \rho^{\mu}\left(t, \rho_{0}\right), t}\right)$, то последовательность чистых состолний $\left\{\rho_{v_{0}^{m}}\right\}$ (или $\left.\left\{\rho_{v_{1}^{m}}\right\}\right)$ сходится в пространстве $B^{*}(H) \kappa$ начальному состолния $\rho_{0}$, причём для определения начального состояния с заданной точностъю достаточно решить конечное число вариачионных задач.

Если вектор $u_{0}$ есть линейная комбинация $u_{00}(t)+u_{01}(t)$ векторов из подпространств $H_{0}(t)$ и $H_{1}(t)$ соответственно, а оператор $\mathbf{A} \in B(H)$, то справедливо равенство

$$
\begin{aligned}
\left\langle\rho^{\mu}\left(t, u_{0}\right), \mathbf{A}\right\rangle=\int_{E}\left(u_{\varepsilon}\left(t, u_{0}\right), \mathbf{A} u_{\varepsilon}\left(t, u_{0}\right)\right) d \mu & \\
=\int_{E}\left[\left(u_{\varepsilon}\left(t, u_{00}\right), \mathbf{A} u_{\varepsilon}\left(t, u_{00}\right)\right)+\right. & \left(u_{\varepsilon}\left(t, u_{01}\right), \mathbf{A} u_{\varepsilon}\left(t, u_{01}\right)\right)+ \\
& \left.+2 \operatorname{Re}\left(u_{\varepsilon}\left(t, u_{01}\right), \mathbf{A} u_{\varepsilon}\left(t, u_{00}\right)\right)\right] d \mu .
\end{aligned}
$$

Согласно предложению 1 , во-первых, предел $\mathbf{A} u_{\varepsilon}\left(t, u_{00}\right)$ при $\varepsilon \rightarrow 0$ существует и равен $\mathbf{A} \mathbf{U}_{-\mathbf{L}}(t)^{*} u_{00}: \lim _{\varepsilon \rightarrow 0}\left\|\mathbf{A} u_{\varepsilon}\left(t, u_{00}\right)-\mathbf{A} \mathbf{U}_{-\mathbf{L}}(t)^{*} u_{00}\right\|$. Во-вторых, в силу 
слабой в пространстве $H$ сходимости к нулю последовательности векторов $\left\{u_{\varepsilon}\left(t, u_{01}\right), \varepsilon \in E, \varepsilon \rightarrow 0\right\}$, справедливо равенство

$$
\lim _{\varepsilon \rightarrow 0}\left(u_{\varepsilon}\left(t, u_{01}\right), \mathbf{A} u_{\varepsilon}\left(t, u_{00}\right)\right)=0 .
$$

Следовательно, $\int_{E}\left(u_{\varepsilon}\left(t, u_{01}\right), \mathbf{A} u_{\varepsilon}\left(t, u_{00}\right)\right) d \mu=0$ и

$$
\begin{aligned}
\left\langle\rho^{\mu}\left(t, u_{0}\right), \mathbf{A}\right\rangle=\int_{E}\left(u_{\varepsilon}\left(t, u_{00}\right), \mathbf{A} u_{\varepsilon}(t,\right. & \left.\left.u_{00}\right)\right) d \mu+ \\
& +\int_{E}\left(u_{\varepsilon}\left(t, u_{01}\right), \mathbf{A} u_{\varepsilon}\left(t, u_{01}\right)\right) d \mu .
\end{aligned}
$$

Итак, получено следующее утверждение о неразличимости начальных состояний с помощью измерений средних значений наблюдаемых (15) в один момент времени.

ПредлоЖение 2. Пусть $t>0$ и вектор состояния $u_{0} \in S^{1}(H)$ имеет проекчии $u_{00}$ и $u_{01}$ на подпространства $H_{0}(t)$ и $H_{1}(t)$ соответственно. Тогда для любых $\mu \in W(E)$ функииональ $\rho^{\mu}\left(t, u_{00}+e^{i \alpha} u_{01}\right)$ совпадают при всех $\alpha \in[0,2 \pi]$.

Лемма 5. Пусть $\rho_{0}=\rho_{u_{0}} \in \Sigma_{p}(H)$. Тогда если последовательность $\left\{v_{0}^{m}\right\}$ единичных векторов пространства $H_{0}(t)$ удовлетворяет равенству

$\sup \varphi_{0}^{m, \rho^{\mu}\left(t, \rho_{0}\right), t}(v)=\varphi_{0}^{m, \rho^{\mu}\left(t, \rho_{0}\right), t}\left(v_{0}^{m}\right)$ при каждом $m \in \mathbb{N}$, то после$v \in S^{1}\left(H_{0}^{m}(t)\right)$

довательность $\left\{v_{0}^{m}\right\}$ компактна в $H$, а любой её частичный предел есть единичный вектор из одномерной линейной оболочки проекции и ио вектора $u_{0}$ на подпространство $H_{0}(t)$.

Дока з а тель ст в о. При фиксированном $m$ супремум непрерывного на компакте $S^{1}\left(H_{0}^{m}(t)\right)$ функционала $\varphi_{0}^{m, \rho^{\mu}\left(t, \rho_{0}\right), t}$ достигается на векторе $v_{m}^{0} \in$ $H_{m}^{0}$. Пусть $\rho_{0}=\rho_{u_{0}}$ и вектор $u_{0}$ имеет проекции $u_{0 s}$ на подпространства $H_{s}(t)$, $s \in\{0,1\}$. Если $s=0$ и $\varphi_{0}^{m, \rho^{\mu}\left(t, \rho_{0}\right), t}(v)=\left\langle\rho^{\mu}\left(t, \rho_{0}\right), \mathbf{P}_{\mathbf{U}_{\mathbf{L}^{*}}(t) v}\right\rangle$, то, согласно (15)

$$
\begin{aligned}
\varphi_{0}^{m, \rho^{\mu}\left(t, \rho_{0}\right), t}(v)=\int_{E}\left(u_{\varepsilon}\left(t, u_{00}\right), \mathbf{P}_{\mathbf{U}_{\mathbf{L}^{*}}(t) v} u_{\varepsilon}\left(t, u_{00}\right)\right) d \mu+ \\
\quad+\int_{E}\left(u_{\varepsilon}\left(t, u_{01}\right), \mathbf{P}_{\mathbf{U}_{\mathbf{L}^{*}}(t) v} u_{\varepsilon}\left(t, u_{01}\right)\right) d \mu
\end{aligned}
$$

следовательно,

$$
\varphi_{0}^{m, \rho^{\mu}\left(t, \rho_{0}\right), t}(v)=\int_{E}\left|\left(\mathbf{U}_{\mathbf{L}^{*}}(t) v, u_{\varepsilon}\left(t, u_{00}\right)\right)\right|^{2} d \mu+\int_{E}\left|\left(\mathbf{U}_{\mathbf{L}^{*}}(t) v, u_{\varepsilon}\left(t, u_{01}\right)\right)\right|^{2} d \mu .
$$

Согласно предложению 1 последовательность функций $u_{\varepsilon}\left(t, u_{00}\right), \varepsilon \in E$, сходится по норме пространства $H$ при $\varepsilon \rightarrow 0$ к вектору $\mathbf{U}_{\mathbf{L}^{*}}(t) u_{00}$, а функция 
$u_{\varepsilon}\left(t, u_{01}\right)$ слабо сходися к нулю при $\varepsilon \rightarrow 0$. Поэтому в силу выбора меры $\mu$ справедливо равенство $\varphi_{0}^{m, \rho^{\mu}\left(t, \rho_{0}\right), t}(v)=\left|\left(v, u_{00}\right)\right|^{2}$ при любом $v$ из области определения $S_{1}\left(H_{0}^{m}(t)\right)$ функционала $\varphi_{0}^{m, \rho^{\mu}\left(t, \rho_{0}\right), t}$. Тогда

$$
\sup _{v_{0} \in S_{1}\left(H_{m}^{0}\right)}\left\langle\rho^{\mu}\left(t, \rho_{u_{0}}\right), \mathbf{P}_{\mathbf{U}_{\mathbf{L}^{*}}(t)} v_{0}\right\rangle=\sup _{v \in S_{1}\left(H_{m}^{0}\right)}\left\|\left(\mathbf{P}_{H_{m}^{0}} u_{0}, v\right)\right\|^{2}=\left\|\mathbf{P}_{H_{m}^{0}} u_{0}\right\|^{2}
$$

достигается на нормированной проекции вектора $u_{0}$ на подпространство $H_{m}^{0}$.

Таким образом, последовательность $\left\{v_{0}^{m}\right\}$ имеет компактное множество значений, а любой ее частичный предел $v_{0}$ коллинеарен проекции вектора $u_{0}$ на подпространство $H_{0}(t)$ и допускает представление $v_{0}=\left(M_{0}(t)\right)^{-\frac{1}{2}} e^{-i \alpha} u_{00}$ при некотором $\alpha \in[0,2 \pi]$.

Пусть выполнены условия леммы 2 и $v_{0}^{*} \in H_{0}(t)$ - один из частичных пределов последовательности элементов $\left\{v_{0}^{m}\right\}$, доставляющих максимум функ-

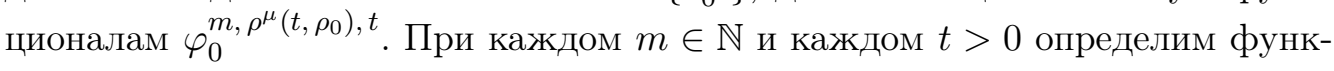
ционал

$$
\begin{aligned}
\psi^{m, \rho^{\mu}\left(t, \rho_{0}\right), t}(v)=\varphi_{1}^{m, \rho^{\mu}\left(t, \rho_{0}\right), t}(v)- \\
\quad-M_{0}(t)\left(\mathbf{U}_{\mathbf{L}^{*}}(t) v_{0}^{*}, \mathbf{Q}_{m}(v) \mathbf{U}_{\mathbf{L}^{*}}(t) v_{0}^{*}\right), \quad v \in S_{1}\left(H_{1}^{m}(t)\right) .
\end{aligned}
$$

Лемма 6. Пусть $\rho_{0}=\rho_{u_{0}} \in \Sigma_{p}(H), s \in\{0,1\}$ и обе проекции вектора $u_{0}$ на подпространства $H_{0}(t)$ и $H_{1}(t)$ нетривиальнв. Пусть и $v_{0} \in H_{0}(t)-$ один из частичных пределов последовательности элементов $\left\{v_{0}^{m}\right\}$, доставляющих максимум функционалам $\varphi_{0}^{m, \rho^{\mu}\left(t, \rho_{0}\right), t}$.

Тогда при каљждых $t>0 u m \in \mathbb{N}$ функиионал $\psi^{m, \rho^{\mu}\left(t, \rho_{0}\right), t}$ не зависит от выбора частичного предела $v_{0}^{*}$ и достигает максимума $M^{m}(t)$ в некоторой точке $v_{1}^{m} \in S_{1}\left(H_{1}^{m}(t)\right)$. При этом последовательность $\left\{M^{m}(t)\right\}$ сходится и её предел $M(t)$ удовлетворлет равенству $M(t)=1-M_{0}(t)$, а последовательность $\left\{v_{1}^{m}\right\}$ компактна в пространстве $H$ и любой её частичный предел $v_{1}^{*}$ допускает представление $v_{1}^{*}=(M(t))^{-\frac{1}{2}} e^{-i \beta} u_{01}$ при некотором $\beta \in[0,2 \pi]$.

Доказ атель ств в. Согласно лемме 5 любой частичный предел $v_{0}$ последовательности $\left\{v_{0}^{m}\right\}$ коллинеарен проекции вектора $u_{0}$ на подпространство $H_{0}(t)$ и допускает представление $v_{0}=\left(M_{0}(t)\right)^{-\frac{1}{2}} e^{-i \alpha} u_{00}$ при некотором $\alpha \in[0,2 \pi]$. Поэтому при любых $m \in \mathbb{N}$ и $t>0$ функционал $\psi^{m, \rho^{\mu}\left(\rho_{0}\right), t}$ не зависит от выбора частичного предела $v_{0}^{*}$ и является непрерывным на компакте $S_{1}\left(H_{1}^{m}(t)\right)$

$$
\begin{aligned}
& \text { Поскольку } \varphi_{1}^{m, \rho^{\mu}\left(t, \rho_{0}\right), t}(v)=\left\langle\rho^{\mu}\left(t, \rho_{0}\right), \mathbf{Q}_{m}(v)\right\rangle, \text { согласно (15) } \\
& \begin{aligned}
\varphi_{1}^{m, \rho^{\mu}\left(t, \rho_{0}\right), t}(v)=\int_{E}\left(u_{\varepsilon}\left(t, u_{00}\right), \mathbf{Q}_{m}(v)\right. & \left.u_{\varepsilon}\left(t, u_{00}\right)\right) d \mu+ \\
& \quad+\int_{E}\left(u_{\varepsilon}\left(t, u_{01}\right), \mathbf{Q}_{m}(v) u_{\varepsilon}\left(t, u_{01}\right)\right) d \mu .
\end{aligned}
\end{aligned}
$$


Таким образом, для любого чистого состояния $\rho_{0} \in \Sigma_{p}(H)$ справедливо равенство

$$
\begin{aligned}
\varphi_{1}^{m, \rho^{\mu}\left(t, \rho_{0}\right), t}(v)=\left\langle\rho^{\mu}\left(t, \mathbf{P}_{H_{0}(t)} \rho_{0} \mathbf{P}_{H_{0}(t)}\right)\right. & \left., \mathbf{Q}_{m}(v)\right\rangle+ \\
& +\left\langle\rho^{\mu}\left(t, \mathbf{P}_{H_{1}(t)} \rho_{0} \mathbf{P}_{H_{1}(t)}\right), \mathbf{Q}_{m}(v)\right\rangle .
\end{aligned}
$$

Замечание. Формула (16), справедливая для чистых начальных состояний $\rho_{0}$, справедлива и для их выпуклых комбинаций и, следовательно, для нормальных состояний. имеем

Тогда с учётом указанной сходимости $u_{\varepsilon}\left(t, u_{00}\right)$ к $\mathbf{U}_{\mathbf{L}^{*}}(t) u_{00}$ при $\varepsilon \rightarrow 0$ $\varphi_{1}^{m, \rho^{\mu}\left(t, \rho_{0}\right), t}(v)=\left(\mathbf{U}_{\mathbf{L}^{*}}(t) u_{00}, \mathbf{Q}_{m}(v) \mathbf{U}_{\mathbf{L}^{*}}(t) u_{00}\right)+\int_{E}\left(u_{\varepsilon}\left(t, u_{01}\right), \mathbf{Q}_{m}(v) u_{\varepsilon}\left(t, u_{01}\right)\right) d \mu$.

Следовательно,

$$
\begin{aligned}
\varphi_{1}^{m, \rho^{\mu}\left(t, \rho_{0}\right), t}(v)-M_{0}(t)\left(\mathbf{U}_{\mathbf{L}^{*}}(t) v_{0}, \mathbf{Q}_{m}(v)\right. & \left.\mathbf{U}_{\mathbf{L}^{*}}(t) v_{0}\right)= \\
& =\int_{E}\left(\mathbf{U}_{\mathbf{L}_{\varepsilon}(t)} u_{01}, \mathbf{Q}_{m}(v) \mathbf{U}_{\mathbf{L}_{\varepsilon}(t)} u_{01}\right) d \mu .
\end{aligned}
$$

Функционал

$$
\int_{E}\left(\mathbf{U}_{\mathbf{L}_{\varepsilon}(t)} u_{01}, \mathbf{Q}_{m}(v) \mathbf{U}_{\mathbf{L}_{\varepsilon}(t)} u_{01}\right) d \mu
$$

исследован в работе [17], согласно лемме 4.3 которой последовательность

$\sup _{\langle\mu}\left\langle\rho^{\mu}\left(t, \rho_{u_{01}}\right), \mathbf{Q}_{v_{1}}(m)\right\rangle, m \in \mathbb{N}$, стремится к $\left\|\mathbf{P}_{H_{1}(t)} u_{0}\right\|^{2}$ при $m \rightarrow \infty$, $v_{1} \in S_{1}\left(H_{m}^{1}\right)$

а последовательность максимизирующих элементов компактна, причём любой её частичный предел коллинеарен вектору $u_{01}$ и допускает представление $v_{1}=(M(t))^{-\frac{1}{2}} e^{-i \beta} u_{01}$ при некотором $\beta \in[0,2 \pi]$.

Из лемм 5 и 6 следует, что если состояние $\rho_{0} \in \Sigma^{p}(H)$, то существуют пределы

$$
\lim _{m \rightarrow \infty} \sup _{v \in S_{1}\left(H_{0}^{m}(t)\right)} \varphi_{0}^{m, \rho^{\mu}\left(t, \rho_{0}\right), t}(v)=M_{0}(t), \lim _{m \rightarrow \infty} \sup _{v \in S_{1}\left(H_{1}^{m}(t)\right)} \psi^{m, \rho^{\mu}\left(t, \rho_{0}\right), t}(v)=M(t)
$$

и выполняется равенство $M_{0}(t)+M(t)=1$.

Согласно лемме 6 равенство $M_{0}(t)+M(t)=1$ необходимо для включения $\rho_{0} \in \Sigma_{p}(H)$, но недостаточно, ибо если $v_{0} \in S_{1}\left(H_{0}(t)\right)$ и $v_{1} \in S_{1}\left(H_{1}(t)\right)$, то для смешанного начального состояния $\rho_{0}=M_{0}(t) \rho_{v_{0}}+M(t) \rho_{v_{1}}$ выполнены равенства $\lim _{m \rightarrow \infty} \sup \left(\varphi_{0}^{t, m, \rho^{\mu}\left(\rho_{0}\right), t}(v)\right)=M_{0}(t)$ и $\lim _{m \rightarrow \infty} \sup \left(\psi^{m, \rho^{\mu}\left(t, \rho_{0}\right), t}(v)\right)=M(t)$. Действительно, для любого $\mathbf{A} \in B(H)$ выполняется равенство $\left\langle\rho^{\mu}\left(t, \rho_{0}\right), \mathbf{A}\right\rangle=$ $=M_{0}(t)\left\langle\rho^{\mu}\left(t, \rho_{v_{0}}\right), \mathbf{A}\right\rangle+M(t)\left\langle\rho^{\mu}\left(t, \rho_{v_{1}}\right), \mathbf{A}\right\rangle$, тогда требуемое равенство следует из (8) и (12).

Покажем, что для однозначного определения динамики математического ожидания $\rho^{\mu}\left(t, \rho_{u_{0}}\right)$ процесса $\rho_{\varepsilon}\left(t, \rho_{u_{0}}\right)$ с чистым начальным состоянием 
$\rho_{u_{0}}$ достаточно знать значения математического ожидания в два различных момента времени.

Если состояние $\rho_{0}$ является чистым - является ортогональным проектором на единичный вектор $u_{0} \in S_{1}(H)$, то тогда для проекций $u_{00}, u_{01}$ вектора $u_{0}$ на подпространства $H_{0}(t), H_{1}(t)$ при любом значении $t>0$ имеют место представления

$$
\mathbf{U}_{-\mathbf{L}}(t) \mathbf{U}_{\mathbf{L}^{*}}(t) u_{0}=u_{00}(t), \quad\left(\mathbf{I}-\mathbf{U}_{-\mathbf{L}}(t) \mathbf{U}_{\mathbf{L}^{*}}(t)\right) u_{0}=u_{01}(t)
$$

и справедливы равенства

$$
M_{0}(t)=\left\|\mathbf{U}_{-\mathbf{L}}(t) \mathbf{U}_{\mathbf{L}^{*}}(t) u_{0}\right\|_{H}^{2}, \quad M(t)=1-M_{0}(t)
$$

Пусть при заданном $t>0$ значения $M_{0}(t), M(t)$ удовлетворяют равенству $M_{0}(t)+M(t)=1$ и элементы $v_{0}^{*}$ и $v_{1}^{*}$ - частичные пределы последовательностей $\left\{v_{0}^{m}\right\}$ и $\left\{v_{1}^{m}\right\}$ из лемм 5 и 6 . Если $M_{0}(t)=0$ (либо $\left.M(t)=0\right)$, то тогда начальный вектор $u_{0}$ целиком лежит в подпространстве $H_{1}(t)$ (либо $H_{0}(t)$ ) и, согласно теореме 2 , начальное состояние $\rho_{u_{0}}$ совпадает с $\rho_{v_{1}^{*}}$ (либо с $\left.\rho_{v_{0}^{*}}\right)$. Исследуем случай, когда для выбранного момента времени $t>0$ выполняется соотношение $M_{0}(t) \in(0,1)$. Положим $H_{0}=\bigcap_{\tau>0} H_{0}(\tau)$, определим по вектору $v_{0}^{*}(t) \in H_{0}(t)$ число

$$
T^{*}=\inf \left\{s \geqslant t: \mathbf{U}_{\mathbf{L}^{*}}(s-t) v_{0}(t) \in H_{0}\right\}
$$

и положим $T^{*}=+\infty$, если $\mathbf{U}_{\mathbf{L}^{*}}(s) v_{0}(t) \notin H_{0}$ при любом $s>0$.

Поскольку полугруппы $\mathbf{U}_{-\mathbf{L}}(t)$ и $\mathbf{U}_{\mathbf{L}^{*}}(t)$ являются непрерывными в сильной операторной топологии, числовая функция $M_{0}(t)=\left\|\mathbf{P}_{H_{0}(t)} u_{0}\right\|_{H}^{2}$ непрерывна на полуоси $t>0$ и монотонно убывает до значения $\left\|\mathbf{P}_{H_{0}} u_{0}\right\|_{H}^{2}$ при $t \rightarrow+\infty$, в частности, является постоянной на интервале $\left(T^{*},+\infty\right)$. Тогда существует такое $t_{1} \in\left(0, T^{*}\right)$, что $M_{0}\left(t_{1}\right) \neq M_{0}(t)$ (см. предложение 1 , а также [7]).

Пусть при заданном $t>0$ определены значения $M_{0}(t), M_{1}(t)$ и частичные пределы $v_{0}(t)$ и $v_{1}(t)$ последовательностей максимизирующих элементов $\left\{v_{0}^{m}\right\}$ и $\left\{v_{1}^{m}\right\}$ (см. леммы 5 и 6), и, кроме того, выполнено равенство $M_{0}(t)+M(t)=1$.

Пусть $\nu$ - мера, существование которой установлено леммой 4. Тогда для каждого числа $t_{j}, j \in\{0,1,2, \ldots\}$, и меры $\nu$ справедливо утверждение лемм 5 и 6. Поэтому, если $\rho_{0} \in \Sigma_{p}(H)$, то существуют частичные пределы $v_{0}^{*}\left(t_{j}\right)$, $v_{1}^{*}\left(t_{j}\right)$ последовательностей, элементы которых реализуют максимумы функционалов $\varphi_{0}^{m, \rho^{\nu}\left(t_{j}, \rho_{0}\right), t_{j}}, \psi^{m, \rho^{\nu}\left(t_{j}, \rho_{0}\right), t_{j}}$ соответственно и существуют пределы $M_{0}\left(t_{j}\right)=\lim _{m \rightarrow \infty} M_{0}^{m}\left(t_{j}\right)$ и $M\left(t_{j}\right)=\lim _{m \rightarrow \infty} M^{m}\left(t_{j}\right)$.

Согласно лемме 6 , для того чтобы состояние $\rho_{0}$ было чистым, необходимо, чтобы $M_{0}\left(t_{j}\right)+M\left(t_{j}\right)=1$. Установим, при каком выборе значения $t_{1} \in(0, T)$ это условие является и достаточным.

В пространстве $B^{*}(H)$ определим кривые

$$
\mathcal{C}\left(t_{j}\right)=\left\{\rho_{\left(M_{0}\left(t_{j}\right)\right)^{\frac{1}{2}} v_{0}^{*}\left(t_{j}\right)+e^{i \alpha}\left(M\left(t_{j}\right)\right)^{\frac{1}{2}} v_{1}^{*}\left(t_{j}\right)}, \alpha \in[0,2 \pi)\right\}, \quad j \in \mathbb{N} .
$$


Лемма 7. Пусть $\rho_{0} \in \Sigma_{n}(H) u t>0$, nусть $M_{0}(t)+M(t)=1 u v_{0}^{*}$, $v_{1}^{*}$-частичные предель максимизирующих последовательностей $v_{0}^{m} u v_{1}^{m}$ из лемм 5 и 6. Пусть, кроме того,

$$
\lim _{m \rightarrow \infty} \sup _{v \in S_{1}\left(H_{0}(t)\right)}\left[\varphi_{0}^{m, \rho^{\mu}\left(t, \rho_{0}\right), t}(v)-M_{0}\left|\left(v_{0}^{*}, v\right)\right|^{2}\right]=0 .
$$

Тогда $\left.\rho_{0}\right|_{B\left(H_{0}(t)\right)}=\left.M_{0}(t) \rho_{v_{0}^{*}} u \rho_{0}\right|_{B\left(H_{1}(t)\right)}=M(t) \rho_{v_{1}}$.

Доказательство. Действительно, согласно $(12)\left(\rho\left(t, \rho_{0}\right), \mathbf{P}_{\mathbf{U}_{\mathbf{L}^{*}}(t) h}\right)=$ $=\left\langle\rho_{0}, \mathbf{P}_{h}\right\rangle$ для любого $h \in S_{1}\left(H_{0}(t)\right)$ и согласно определению вектора $v_{0}^{*}$ справедливо равенство $\left(\rho\left(t, \rho_{0}\right), \mathbf{P}_{\mathbf{U}_{\mathbf{L}^{*}}(t) v_{0}^{*}}\right)=M_{0}(t)$.

Оператор $\mathbf{P}_{H_{0}(t)} \rho_{0} \mathbf{P}_{H_{0}(t)}$ есть неотрицательный оператор с конечным следом в пространстве $H$, ядро которого содержит подпространство $H_{1}(t)$. По свойству экстремальности собственных значений компактного оператора число $M_{0}(t)$ есть максимальное собственное значение оператора $\mathbf{P}_{H_{0}(t)} \rho_{0} \mathbf{P}_{H_{0}(t)}$, a $v_{0}^{*}$ - соответствующий ему единичный собственный вектор. Поэтому функционалы $\rho_{0}$ и $M_{0}(t) \mathbf{P}_{v_{0}}$ совпадают на одномерном подпространстве $\operatorname{span}\left(v_{0}\right)$ и $\rho_{0}-M_{0}(t) \rho_{v_{0}}$ - неотрицательный функционал на $B(H)$. Но поскольку

$$
\varphi_{0}^{m, \rho_{0}-M_{0}(t) \rho_{v_{0}}, t}(v)=\varphi_{0}^{m, \rho^{\mu}\left(t, \rho_{0}\right), t}(v)-M_{0}\left|\left(v_{0}, v\right)\right|^{2},
$$

в силу условия леммы

$$
\lim _{m \rightarrow \infty} \sup _{v \in S_{1}\left(H_{0}(t)\right)}\left[\varphi_{0}^{m, \rho^{\mu}\left(t, \rho_{0}\right), t}(v)-M_{0}\left|\left(v_{0}^{*}, v\right)\right|^{2}\right]=0
$$

выполняется равенство $\left.\rho_{0}\right|_{B\left(H_{0}(t)\right)}=M_{0}(t) \rho_{v_{0}}$. Следовательно, для векторов $h \in S_{1}\left(H_{1}^{m}(t)\right)$ в силу равенства $(16)$ и замечания к нему справедливо соотношение

$\varphi_{1}^{m, \rho^{\mu}\left(t, \rho_{0}\right), t}(h)=\left\langle\rho^{\mu}\left(t, \mathbf{P}_{H_{0}(t)} \rho_{0} \mathbf{P}_{H_{0}(t)}\right), \mathbf{Q}_{m}(h)\right\rangle+\left\langle\rho^{\mu}\left(t, \mathbf{P}_{H_{1}(t)} \rho_{0} \mathbf{P}_{H_{1}(t)}\right), \mathbf{Q}_{m}(h)\right\rangle$.

Согласно доказанному соотношению $\left.\rho_{0}\right|_{B\left(H_{0}(t)\right)}=M_{0}(t) \rho_{v_{0}^{*}}$ значения функционала

$$
\begin{aligned}
\psi^{m, \rho^{\mu}\left(t, \rho_{0}\right), t}(h)=\varphi_{1}^{m, \rho^{\mu}\left(t, \rho_{0}\right), t}(h)-M_{0}(t)\left\langle\rho_{\mathbf{U}_{\mathbf{L}^{*}(t) v_{0}^{*}}}, \mathbf{Q}_{m}(v)\right\rangle= \\
\quad=\left\langle\rho^{\mu}\left(t, \mathbf{P}_{H_{1}(t)} \rho_{0} \mathbf{P}_{H_{1}(t)}\right), \mathbf{Q}_{m}(h)\right\rangle
\end{aligned}
$$

стремятся (см. лемму $4.3[17])$ при $m \rightarrow \infty$ к значению $\left\langle\mathbf{P}_{H_{1}(t)} \rho_{0} \mathbf{P}_{H_{1}(t)}, \mathbf{P} h\right\rangle=$ $=\left\langle\rho_{0}, \mathbf{P}_{h}\right\rangle$.

Оператор $\mathbf{P}_{H_{1}(t)} \rho_{1} \mathbf{P}_{H_{0}(t)}$ есть неотрицательный оператор с конечным следом в пространстве $H$, ядро которого содержит подпространство $H_{0}(t)$. По свойству экстремальности собственных значений компактного оператора число $M(t)$ есть максимальное собственное значение оператора $\mathbf{P}_{H_{1}(t)} \rho_{0} \mathbf{P}_{H_{1}(t)}$, a $v_{1}^{*}$ - соответствующий ему единичный собственный вектор.

Поэтому функционал $\rho_{0}$ мажорирует функционал $M(t) \rho_{v_{1}^{*}}$, совпадая с ним на одномерном подпространстве $\operatorname{span}\left(v_{1}^{*}\right)$. Следовательно, $\left\|\left.\rho_{0}\right|_{B\left(H_{1}\right)}\right\|_{B^{*}(H)} \geqslant M_{1}$. 
Значит, состояние $\rho_{0} \in \Sigma_{n}(H)$ мажорирует функционал $M_{0}(t) \rho_{v_{0}^{*}}+M(t) \rho_{v_{1}^{*}}$. Поскольку $\rho_{0} \in \Sigma(H)$ и $M_{0}(t)+M(t)=1$, можно записать, что $\rho_{0}=M_{0}(t) \rho_{v_{0}^{*}}+$ $+M(t) \rho_{v_{1}^{*}}$.

СледствиЕ 2. Пусть выполнены условия леммы 7. Тогда

1) если состояние $\rho_{0}$ не является чистым, то оно является смесъю: выпуклой комбинацией двух чистых состояний $\rho_{0}=M_{0}(t) \rho_{v_{0}^{*}}+M(t) \rho_{v_{1}^{*}}$;

2) если состояние $\rho_{0}$ чистое с вектором состояния $u_{0} \in S_{1}(H)$, то проекиии вектора $u_{0}$ на подпространства $H_{0}(t)$ и $H_{1}(t)$ есть соответственно $v_{0}^{*} e^{i \alpha} u v_{1}^{*} e^{i \beta}$ с некоторыми фазами $\alpha, \beta \in[0,2 \pi]$.

Доказ а тель ст в о. Нормальное состояние $\rho_{0}$ допускает представление в виде

$$
\rho_{0}=\sum_{k=1}^{\infty} p_{k} \rho_{e_{k}},
$$

где $\left\{e_{k}\right\}$-ортонормированный базис в $H, p_{k} \in[0,1], k \in \mathbf{N}$. Сужения функционала $\rho_{0}$ на $B\left(H_{0}(t)\right)$ и $B\left(H_{1}(t)\right)$ есть $\left.\rho_{0}\right|_{B\left(H_{s}\right)}=\mathbf{P}_{H_{s}(t)} \rho_{0} \mathbf{P}_{H_{s}(t)}$, согласно условиям леммы 7 функционалы $\left.\rho_{0}\right|_{B\left(H_{0}(t)\right)},\left.\rho_{0}\right|_{B\left(H_{1}(t)\right)}$ оба кратны чистому состоянию, поэтому либо состояние $\rho_{0}$ чистое и справедливо второе утверждение следствия, либо ортогональное разложение следового оператора $\rho_{0}$ коммутирует с проектором $\mathbf{P}_{H_{0}(t)}$ и справедливо первое утверждение следствия.

Пусть $t>0, \mu$-мера, существование которой доказано в лемме 3 и число $t_{1} \in\left(0, T^{*}\right) \backslash\{t\}$ рационально соизмеримо с числом $t$.

Тогда согласно леммам 5 и 6 существуют единичные векторы $v_{0}^{*}\left(t_{1}\right), v_{1}^{*}\left(t_{1}\right)-$ частичные пределы последовательностей, элементы которых реализуют максимумы $M_{0}^{m}\left(t_{1}\right), M^{m}\left(t_{1}\right)$ функционалов $\varphi_{0}^{m, \rho^{\nu}\left(t_{1}, \rho_{0}\right), t_{1}}, \psi^{m, \rho^{\nu}\left(t_{1}, \rho_{0}\right), t_{1}}$ соответственно. Для каждого рационального числа $t_{1}>0$ обозначим через $C\left(t_{1}\right)$ замкнутую кривую $\left\{\rho_{\left(M_{0}\left(t_{1}\right)\right)^{\frac{1}{2}} v_{0}^{*}\left(t_{1}\right)+e^{i \beta}\left(M\left(t_{1}\right)\right)^{\frac{1}{2}} v_{1}^{*}\left(t_{1}\right)}, \beta \in[0,2 \pi)\right\}$ в пространстве квантовых состояний.

Теорема 5. Пусть $t>0$ и $\rho_{0}-$ нормальное состояние.

1. Если состояние $\rho_{0}$ чистое, то выполнены равенства $M_{0}(t)+M(t)=1$, $\lim _{m \rightarrow \infty} \sup _{v \in S_{1}\left(H_{0}(t)\right)}\left[\varphi_{0}^{m, \rho^{\mu}\left(t, \rho_{0}\right), t}(v)-M_{0}(t)\left|\left(v_{0}, v\right)\right|^{2}\right]=0$ и включение $\rho_{0} \in \mathcal{C}(t)$.

2. Если $M_{0}(t)=0$ или $M_{0}(t)=1$, то состолние $\rho_{0}$ чистое, а кривал $\mathcal{C}(t)$ cостоит из одной точки.

3. Если равенство $M_{0}(t)+M(t)=1$ выполнено и $M_{0}(t) \in(0,1)$, то существует такое рачионально соизмеримое с $t$ число $t_{j} \in\left(0, T^{*}\right)$, что $M_{0}(t) \neq M_{0}\left(t_{j}\right)$. Кроме того, пусть

$$
\lim _{m \rightarrow \infty} \sup _{v \in S_{1}\left(H_{0}(t)\right)}\left[\varphi_{0}^{m, \rho^{\mu}\left(t, \rho_{0}\right), t}(v)-M_{0}(t)\left|\left(v_{0}, v\right)\right|^{2}\right]=0 .
$$

Тогда для включения $\rho_{0} \in \Sigma_{p}(H)$ достаточно, чтобы $M_{0}\left(t_{j}\right)+M\left(t_{j}\right)=1$. $B$ этом случае кривые $\mathcal{C}(t)$ и $\mathcal{C}\left(t_{j}\right)$ имеют единственную общую точку, которая и является начальным состоянием $\rho_{0}$. 
Доказательство. Необходимость для включения $\rho_{0} \in \Sigma_{p}(H)$ и равенства $M_{0}(t)+M(t)=1$ следует из леммы 6 , а включения $\rho_{0} \in \mathcal{C}(t)$-из второй части следствия 2 .

Из условия $\max \left(M_{0}(t)=1\right.$ (либо $\left.M_{0}(t)\right)=0$ ) и теоремы 2 следует, что состояние $\rho_{0}$ чистое, а кривая $\mathcal{C}(t)$ состоит из одной точки $\rho_{0}$.

Существование такого рационального числа $t_{1} \in\left(0, T^{*}\right)$, что $M_{0}\left(t_{1}\right) \neq M_{0}(t)$, следует из непрерывности функции $M_{0}(t)$ согласно соотношению (17) и условия $M_{0}(0)=1$. Тогда из доказанного утверждения 1 теоремы 5 следует, что условия $M_{0}\left(t_{1}\right)+M\left(t_{1}\right)=1$ и $\rho_{0} \in \mathcal{C}\left(t_{1}\right)$ необходимы для включения $\rho_{0} \in \Sigma_{p}$. Предположим, что $\rho_{0} \in \Sigma_{n} \backslash \Sigma_{p}$, тогда справедлива первая часть утверждения следствия 2 для момента времени $t$, следовательно, состояние $\rho_{0}$ должно быть представимо в каждом из следующих двух видов:

$$
\rho_{0}=M_{0}(t) \rho_{v_{0}^{*}(t)}+M(t) \rho_{v_{1}^{*}(t)}=M_{0}\left(t_{1}\right) \rho_{v_{0}^{*}\left(t_{1}\right)}+M\left(t_{1}\right) \rho_{v_{1}^{*}\left(t_{1}\right)} .
$$

Последнее равенство невозможно при $M_{0}(t) \neq M_{0}\left(t_{1}\right)$. Полученное противоречие доказывает, что состояние $\rho_{0}$ чистое и принадлежит $\mathcal{C}(t) \cap \mathcal{C}\left(t_{1}\right)$. Проекции начального вектора состояния $u_{0} \in S_{1}(H)$ на пары подпространств $H_{0}(t), H_{1}(t)$ и $H_{0}\left(t_{1}\right), H_{1}\left(t_{1}\right)$ связаны соотношением $(17)$, что при условии $M_{0}\left(t_{1}\right) \in\left(M_{0}(t), 1\right)$ позволяет определить начальный вектор $u_{0}$ по его проекциям на указанные подпространства однозначно. Следовательно, каждому чистому начальному состоянию соответствует единственная общая точка кривых $\mathcal{C}(t)$ и $\mathcal{C}\left(t_{1}\right)$.

Итак, в силу предложения 2 и теоремы 5 математическое ожидание $T^{\nu}(t)$ случайного процесса $T_{\varepsilon}(t)$ как функция переменной $t$ не является однопараметрической полугруппой преобразований банахова пространства $B^{*}(H)$. Математическое ожидание случайного процесса $\rho_{\varepsilon}\left(t, \rho_{u_{0}}\right)$ может быть определено из решения вариационных задач.

Работа выполнена при поддержке РФФИ (проекты №o 09-01-00265-a, 10-01-00395-а), ФЦП «Научные и научно-педагогические кадры инновационной России» на 2009-2013 годы и АВЦП «Развитие научного потенциала высшей школы» (проект № 2.1.1/11133).

\section{БИБЛИОГРАФИЧЕСКИЙ СПИСОК}

1. Pavlotsky I.P., Strianese M. Irreversibility in classical mechanics as a consequence of Poincaré group // Inter. J. of Mod. Phys. B., 1996. Vol. 10, no. 21. Pp. 2675-2685.

2. Сакбаев В. Ж. О спектральных аспектах регуляризации задачи Коши для вырожденного уравнения / В сб.: Дифференциалъные уравнения и динамические системы: Сборник статей / Тр. МИАН, Т. 261. М.: МАИК, 2008. С. 258-267; англ. пер.: Sakbaev V. Zh. Spectral aspects of regularization of the Cauchy problem for a degenerate equation // Proc. Steklov Inst. Math., 2008. Vol. 261. Pp. 253-261.

3. Козлов В.В. Динамика систем с неинтегрируемыми связями // Вестн. Моск. ун-та. Cер. 1. Математика. Механика, 1987. №5. C. 76-83. [Kozlov V. V. Dynamics of systems with nonintegrable constraints // Vestn. Mosk. Un-ta. Ser. 1. Matematika. Mekhanika, 1987. no. 5. Pp. 76-83].

4. Accardi L., Lu Y. G., Volovich I. V. Quantum theory and its stochastic limit. Berlin, Heidelberg, New York: Springer-Verlag, 2001. 473 pp.

5. Боголюбов Н. Н. О некоторых статистических методах в математической физике. Киев: Изд-во АН УССР, 1945. 139 с. [Bogoliubov N. N. On some statistical methods in mathematical physics. Kiev: Izd-vo AN USSR, 1945. 139 pp.]

6. Bratteli O., Robinson D. W. Operator Algebras and Quantum Statistical Mechanics. Vol. I: $C^{*}$ - and $W^{*}$-Algebras Symmetry Groups Decomposition of States / Second Edition. Berlin, 
Heidelberg, New York: Springer-Verlag, 2003. 505 pp.; русск. пер.: Брателли У., Робинсон Д. Операторные алгебры и квантовая статистическая механика. М.: Мир, 1982. $512 \mathrm{c}$.

7. Сакбаев В. Ж. О многозначных отображениях, задаваемых регуляризацией уравнения Шрёдингера с вырождением// Ж. вычисл. матем. и матем. физ., 2006. Т. 46, № 4 . C. 683-699; англ. пер.: Sakbaev V. Zh. Set-valued mappings specified by regularization of the Schrödinger equation with degeneration // Comput. Math. Math. Phys., 2006. Vol.46, no. 4. Pp. 651-665.

8. Dunford N., Schwartz J.T. Linear Operators. Vol. 1: General Theory. New York: John Willey and Sons, 1988. 858 рр.; русск. пер.: Данфорд Н., Швари, Д. Теория операторов. Т. 1: Общая теория. М.: УРСС, 2010. 896 с.

9. Yosida K., Hewitt E. Finitely additive measures// Trans. Am. Math. Soc., 1952. T. 72 C. $46-66$.

10. Emch G. G. Algebraic Methods in Statistical Mechanics and Quantum Field Theory/ Physics \& Astronomical Monograph. New York: John Willey and Sons, 1972. 350 pp.; русск. пер.: Эмx Ж. Алгебраические методы в статистической механике и квантовой теории поля. М.: Мир, 1976. 423 с.

11. Glauber R. J. Optical coherence and photon statistics / In: Quantum Optics and Electronics; eds. C. deWitt, A. Blandin, C. Cohen-Tannoudji. New York: Gordon and Breach, 1965. Pp. 65-185; русск. пер.: Глаубер Р. Оптическая когерентность и статистика фотонов / В сб.: Квантовая оптика и квантовая радиофизика. М.: Мир, 1966. С. 93-279.

12. Кери А. Л., Сукочев Ф. А. Следы Диксмье и некоторые приложения в некоммутативной геометрии // УМH, 2006. Т. 61, №6(372). С. 45-110; англ. пер.: Carey A. L., Sukoche F. A. Dixmier traces and some applications in non-commutative geometry// Russian Math. Surveys, 2006. Vol.61, no. 6. Pp. 1039-1099.

13. Srinivas M.D. Collapse postulate for observables with continuous spectra// Commun. Math. Phys., 1980. Vol. 71, no. 2. Pp. 131-158.

14. Сакбаев В. Ж. Об усреднении квантовых динамических полугрупп // ТМФ, 2010. Т. 164, №3. C. 455-463; англ. пер.: Sakbaev V. Zh. Averaging of quantum dynamical semigroups // Theoret. and Math. Phys., 2010. Vol. 164, no. 3. Pp. 1215-1221.

15. Варадарайн В. С. Меры на топологических пространствах // Матем. сб., 1961. Т. 55(97), № 1. C. 35-100. [Varadarajan V.S. Measures on topological spaces // Matem. Sb., 1961. Vol. 55(97), no. 1. Pp. 35-100].

16. Амосов Г. Г., Сакбаев В. Ж. Стохастические свойства динамики квантовых систем // Вестн. Сам. гос. ун-та. Естественнонаучн. сер., 2008. №8/1(67). С. 479-494. [Amosov GC., Sakbaev V.Zh. Stochastic properties of the dynamics of quantum systems // Vestn. Sam. Gos. Un-ta. Estestvennonauchn. Ser., 2008. no.8/1(67). Pp. 479-494].

17. Сакбаев В. Ж. О динамике вырожденной квантовой системы в пространстве функций, интегрируемых по конечно-аддитивной мере // Труды МФТИ, 2009. Т. 1, № 4. С. 126147. [Sakbaev V.Zh. On the dynamics of a degenerate quantum system in the space of integrable functions on a finitely additive measure// Trudy MFTI, 2009. Vol.1, no. 4. Pp. 126-147].

Поступила в редакцию 20/XII/2010;

в окончательном варианте - 03/III/2011. 
MSC: 35Q55; 81Q05

\section{ON THE DYNAMICS OF THE QUANTUM STATES SET FOR} A SYSTEM WITH DEGENERATED HAMILTONIAN

\section{Zh. Sakbaev ${ }^{1,2}$}

1 Moscow Institute of Physics and Technology,

9, Inststitutskiy per., Dolgoprudniy, Moskovskaya obl., 141700, Russia.

2 Peoples Friendship University of Russia,

6, Mikluho-Maklai st., Moscow, 117198, Russia.

E-mail: fumi2003@mail.ru

We study the sequence of regularizing Cauchy problem as the elliptic regularization of Cauchy problem for Schrodinger equation with discontinuous and degenerated coefficients. The necessary and sufficient conditions of the convergence of the regularizing dynamical semigroups sequence are presented. If the convergence is impossible then divergent sequence of the regularizing quantum states is considered as the stochastic process on the measurable space of regularizing parameter endowing with finite additive measure. The expectation of this stochastic process defines the averaging trajectory in the space of quantum states. It was obtained the condition on the finite additive measure such, that averaging trajectory can be defined by its values in two instants with the help of solving the variational problems.

Key words: finite additive measure, stochastic process, quantum state, dynamical semigroup, observability.

Original article submitted 20/XII/2010; revision submitted $03 / \mathrm{III} / 2011$.

Vsevolod Zh. Sakbaev (Dr. Sci. (Phys. \& Math.)), Associate Professor, Dept. of Higher Mathematics ${ }^{1}$; Associate Professor, Dept. of Differential Equation and Mathematical Physics $^{2}$. 